
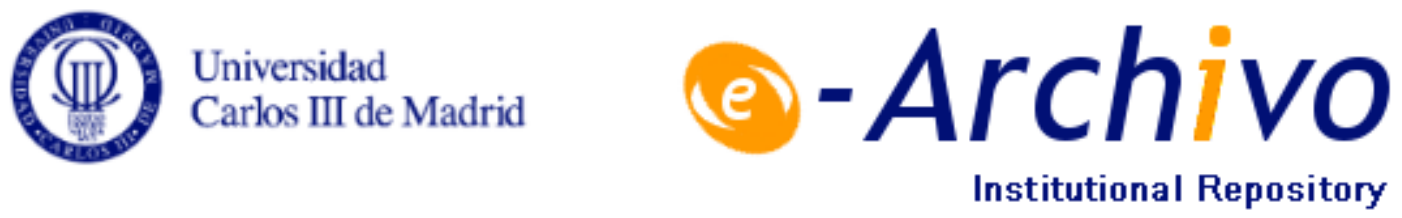

This is a postprint version of the following published document:

Dziuda, W., Loeper, A., (2016), Dynamic Collective Choice with Endogenous Status Quo. Journal of Political Economy , v. 124, n. 4, pp. 1148 -1186. Available in : https://doi.org/10.1086/686747

(C) University of Chicago Press 


\title{
Dynamic Collective Choice with Endogenous Status Quo
}

\section{Wioletta Dziuda}

University of Chicago

\section{Antoine Loeper}

Universidad Carlos III de Madrid

\begin{abstract}
We analyze a bargaining situation in which preferences evolve over time and the previous agreement becomes the next status quo. The endo geneity of the status quo exacerbates the players' conflict of interest: Players disagree more often than under exogenous status quo. This leads to inefficiencies and status quo inertia. Under certain conditions, the negotiations can come to a complete gridlock: Players never reach an agreement. Gridlock can occur between players with arbitrarily sim ilar preferences, provided they are sufficiently patient. In legislative set tings, our model predicts polarization and explains why legislators may fail to react promptly to economic shocks.
\end{abstract}

\section{Introduction}

Despite a wide consensus on the necessity of reforms in important policy areas such as entitlements, immigration laws, or the tax code, the US Con-

This paper previously circulated under the title "Ongoing Negotiation with an Endogenous Status Quo.” We thank David Austen Smith, David Baron, Daniel Diermeier, Bard Harstad, Alessandro Riboni, and seminar participants at Bonn University, Columbia University, Co legio Universitario de Estudios Financieros, Harris School of Public Policy, Leuven Univer sity, the London Business School, Northwestern University, Nottingham University, the Paris Game Theory Seminar, Rice University, Simon Fraser University, Universidad de Alicante, Universidad Carlos III, Universidad Complutense, and the University of Chicago. Yuta Taka hashi provided excellent research assistance. Loeper acknowledges the financial support 
gress has failed to act on these issues. The economic literature has proposed several explanations for the inability of legislators to reach seemingly beneficial agreements such as electoral calculus, vested interests, or uncertainty over the distributional impact of reforms (Drazen 2002). This paper shows that gridlock and legislative inertia can be the strategic consequence of two features of policy making. First, the policies that govern entitlements, immigration, or taxation are typically continuing in nature, remaining in effect until a new agreement is reached. Second, a constantly evolving environment requires these policies to be periodically revisited.

To understand how the combination of the continuing nature of policies and an evolving environment leads to excessive disagreement, consider the case of legislators negotiating over taxation. When the budget surplus is large or the returns to public spending are low, lower tax rates may be favored by all parties to ease the tax burden of the citizens. When deficits run high or the returns to public spending are high, all parties may agree to generate extra tax revenues to reduce the public debt or finance socially desirable programs. In other times, however, legislators may genuinely disagree on whether taxes should be raised or cut. If today's policy becomes the default for future negotiations, liberals may be reluctant to decrease taxes when that is desirable, out of fear that their conservative counterparts will veto tax increases when the need for high taxes arises. Similarly, anticipating future disagreement, conservatives may refuse to increase taxes when needed, out of fear that liberals will oppose tax cuts in the future.

We first formalize this insight in a model in which two players engage in an infinite sequence of choices over two alternatives, called $L$ and $R$. At the beginning of each period, one alternative serves as the status quo. If both players agree to move away from the status quo, the new agreement is implemented. Otherwise, the status quo stays in place. In both cases, the implemented alternative determines the players' payoffs in the present period and becomes the status quo for the next; that is, players operate under the endogenous status quo protocol. We assume that players' preferences over the alternatives change over time. They sometimes disagree, and whenever they do, one player (the rightist) prefers $R$ and the other (the leftist) prefers $L$.

We show that in all stationary equilibria, the leftist player votes for $L$ and the rightist player votes for $R$ more often than they would if the policies were not continuing in nature or if there were no shocks to the preferences. Thus, the endogenous status quo exacerbates players' ideological polarization. As a result, they may fail to reach an agreement even

from the Ministerio Economía y Competitividad (Spain), grants ECO 201342710 P, MDM 2014 0431, and Comunidad de Madrid, MadEco CM (S2015/HUM 3444). 
when the status quo is Pareto dominated. The status quo stays in place too often, and the bargaining outcome is insufficiently responsive to the environment.

Our analysis reveals that players' equilibrium behavior is driven by a vicious cycle: players that behave in a polarized way disagree more often; more frequent disagreement in turn increases players' incentives to secure their preferred status quo and thus increases their incentives to become even more polarized. Because of this vicious cycle, the polarizing effect of the endogenous status quo can be quite dramatic. We identify a class of payoff profiles such that for any profile in that class, if players are sufficiently patient, then the negotiations can come to a complete gridlock in which players never reach an agreement, and the bargaining outcome is unresponsive to the evolution of preferences. Interestingly, this class of payoff profiles allows players' preferences to be arbitrarily similar.

When players bargain over more than two alternatives, the polarizing effect of the endogenous status quo can be accompanied by other equilibrium effects. These effects depend on the fine details of the model such as the process that governs the evolution of the state or the bargaining procedure used to select the outcome in each period (with more than two alternatives, not all bargaining procedures are equivalent). The complex interaction of these effects makes it hard to isolate analytically the impact of the endogenous status quo on the equilibrium behavior. However, we identify two arguably relevant environments in which our results are qualitatively unchanged irrespective of the number of alternatives. In Section IV.B, we analyze a setting in which in each period, players use a bargaining procedure that favors incremental policy changes. Such a procedure captures the incremental nature of legislative policy making (Hayes 1992; Wildavsky 1992) and has been shown to generate predictions consistent with the actual decisions of monetary policy committees (Riboni and Ruge-Murcia 2010). We show that under this procedure, the endogenous status quo generates polarization and status quo inertia as in the case of two alternatives. Moreover, in certain environments, the magnitude of these effects is independent of the space of alternatives. In Section IV.C, we allow for a large class of within-period bargaining procedures, but in contrast to the two-alternative model in which players bargain once every period, we assume that preferences change smoothly and players can revise the status quo as soon as the environment renders it inadequate. This assumption is meant to reflect the fact that actual bargaining institutions typically allow the bargaining parties to propose policy changes whenever the situation prompts it. We show that in such an environment, the equilibrium outcome exhibits status quo inertia.

Our model is institutionally sparse but can shed light on some aspects of policy making in the US Congress. It predicts that in policy areas gov- 
erned by permanent legislations and subject to changing needs-such as mandatory spending, taxation, or civil liberties and national security-legislators' voting behavior can exhibit an excessive degree of polarization that can lead to inefficient policy inertia. Clearly, our model cannot claim to explain all gridlock in Congress nor the dramatic increase in polarization that has been happening over the last decades. It does, however, suggest that the degree of polarization in legislators' voting record may overestimate their actual degree of ideological polarization. We discuss this issue in more detail in Section V.

Our results also have implications for other institutional settings. In some countries, monetary policy is set by a committee with heterogeneous preferences and beliefs, and the interest rate stays the same until the committee agrees to change it (see Riboni and Ruge-Murcia 2008). Our results show that the endogeneity of the status quo can affect the ability of the committee to respond to economic shocks. Similarly, the renegotiations of ongoing contracts such as trade agreements, international treaties (e.g., for the World Trade Organization or the European Union), and financial or labor contracts are usually conducted in the presence of a binding previous agreement. The impossibility of making these contracts fully contingent on all economic shocks and political events creates the need for frequent renegotiation, making the forces we have highlighted relevant in these settings.

\section{Related Literature}

\section{A. Inefficiency and Inertia in Dynamic Policy Making Models}

Policy makers' incentives (not) to adopt socially beneficial policy changes have been the study of numerous papers. Inefficient status quo inertia can arise if there is uncertainty over who benefits from the policy change (Fernandez and Rodrik 1991; Strulovici 2010), if the costs of policy change are borne disproportionately by those who initiate these changes (Alesina and Drazen 1991), or if citizens respond to the current policy by undertaking private actions that make them more likely to oppose a policy change in the future (Glomm and Ravikumar 1995; Krusell and RiosRull 1996; Coate and Morris 1999). In our model, it is neither the uncertainty over future preferences nor their dependence on past policies that matters, but the mere fact that they evolve. ${ }^{1}$

\footnotetext{
${ }^{1}$ Inefficiencies can also arise if the allocation of decision power evolves over time (Pers son and Svensson 1989; Alesina and Tabellini 1990; Krusell and Rios Rull 1999; Battaglini and Coate 2007, 2008; Azzimonti 2011) or if it depends on past decisions (Besley and Coate 1998; Acemoglu and Robinson 2001; Acemoglu, Egorov, and Sonin 2008, 2012, 2015; Bai and Lagunoff 2011; Baron, Diermeier, and Fong 2012; Cho 2012; Duggan and Forand 2013).
} 


\section{B. Bargaining with an Endogenous Status Quo in a Static Environment}

Our paper complements the literature on dynamic bargaining with an endogenous status quo. Most of this literature considers environments in which preferences are static; the bargaining outcome changes over time because the identity of the proposer changes or because the same proposer seeks the support of a different coalition. In our basic model with two alternatives and unanimity rule, these two channels do not play any role. Instead, the impetus for policy change comes solely from the evolution of preferences. In static environments with collective goods policies, this literature (Baron 1996; Baron and Herron 2003; Zapal 2011b) finds that the endogenous status quo has a moderating effect. In contrast, we show that in a changing environment, the endogenous status quo has a polarizing effect.

The moderating effect highlighted by the aforementioned literature is similar to the effect that occurs in this model in the case of more than two alternatives (see Sec. IV.A). This effect is closely related to the observation initially made by Romer and Rosenthal (1978) that an extreme status quo increases the leverage of the proposer and is thus detrimental to nonproposers. Therefore, with an endogenous status quo, implementing a moderate policy today can constrain the next proposer. Diermeier and Fong (2011) show that this effect can limit the bargaining power of a monopolistic proposer, and Bowen, Chen, and Eraslan (2014) show that it mitigates the inefficiency due to political uncertainty. We contribute to that literature by showing that in a stochastic environment, under reasonable conditions, the moderating effect is dominated by the polarizing effect.

Since Kalandrakis (2004), a large literature has looked at the case of purely distributive policies. ${ }^{2}$ These papers focus on the fairness of equilibrium allocations and on the size and stability of the coalitions that support them. By virtue of analyzing collective goods policies under the unanimity rule, our paper has nothing to say on these issues. Instead, we focus on the responsiveness of policies to the environment and on polarization, on which the aforementioned papers are mute, as in the distributive setting, players' preferences are constant and always diametrically opposed.

\section{Bargaining with an Endogenous Status Quo in an Evolving Environment}

Even though dynamic bargaining with an endogenous status quo in a stochastic environment is at the center of many economically relevant situa-

${ }^{2}$ See, among others, Bernheim, Rangel, and Rayo (2006), Anesi (2010), Kalandrakis (2010), Diermeier and Fong (2011), Anesi and Seidmann (2015), Bowen and Zahran (2012), Baron and Bowen (2013), and Richter (2014). 
tions, the existing literature on this topic is scarce. To the best of our knowledge, only Riboni and Ruge-Murcia (2008), Zapal (2011a), Duggan and Kalandrakis (2012), and Bowen, Chen, Eraslan, and Zapal (2014) make progress on this front. Riboni and Ruge-Murcia (2008) solve a two-period, two-state example with quadratic preferences and use numerical solutions for a six-state, infinite-horizon model. Zapal (2011a) considers a two-state environment with quadratic preferences and a continuum of alternatives. These authors recognize that the dynamic linkage may lead to inefficient policies. However, they do not identify the systematic polarizing effect of the endogenous status quo. Adding noise to the status quo, Duggan and Kalandrakis (2012) establish the existence of an equilibrium in a very general setting. Bowen et al. (2014) show that the inefficiencies generated by the endogenous status can be eliminated if the status quo can be fully contingent on the state of nature.

Our paper differs from these contributions in that by focusing on two alternatives, we show that the polarizing and inertial effects arise in every equilibrium for a large class of environments, and we characterize the determinants of their magnitude. We also investigate the conditions under which these effects are robust to allowing for more alternatives.

Riboni and Ruge-Murcia (2010) analyze a game similar to our $\mathrm{N}$ alternative model under four different within-period bargaining procedures. For tractability, they assume myopic players, which severs the strategic link present in our model. One within-period bargaining procedure - the consensus procedure - is shown to lead to least frequent and most moderate policy changes. Using data from five central banks, Riboni and Ruge-Murcia find that all procedures, including the consensus procedure, underestimate the actual degree of status quo inertia. Consistently with this finding, in Section IV.B, we consider the consensus procedure and show that with forward-looking players, the endogenous status quo can dramatically increase status quo inertia.

\section{The Two-Alternative Model}

\section{A. The Model}

Two players, $l$ and $r$, are in a relationship that lasts for infinitely many periods. There are two alternatives, $X=\{L, R\}$. In each period $t$, a status quo $q(t) \in\{L, R\}$ is in place, and players vote simultaneously on which alternative to adopt. If both players vote for the same alternative, this alternative is implemented. If they disagree, this period's status quo $q(t)$ is implemented. The implemented alternative $x(t)$, be it the new agreement or the status quo, determines the players' payoff in $t$ and becomes the status quo for the next period $t+1$. 
The payoff of player $k \in\{l, r\}$ in period $t$ from alternative $x \in X$ is denoted by $U_{k}(\theta(t), x)$, where $\theta(t)$ denotes the state of nature in that period. To capture the dynamic nature of the environment, we assume that $\{\theta(t): t \geq 0\}$ is a stationary Markov process on an arbitrary (finite or infinite) state space $\Theta$ with sigma algebra $\mathcal{S}$. That is, the state $\theta(t)$ depends on the past only through last period's state $\theta\left(\begin{array}{ll}t & 1\end{array}\right)$, and the distribution of $\theta(t)$ conditional on $\theta\left(\begin{array}{ll}t & 1\end{array}\right)$ is independent of $t$. Note that this assumption does not rule out deterministic processes. Throughout, $\theta$ denotes an arbitrary realization of $\theta(t)$, and $P_{\theta}$ denotes the probability distribution of $\theta(t+1)$ conditional on $\theta(t)=\theta$. The state $\theta(t)$ is observed in $t$ by both players before they cast their votes. We assume that for all $x \in X, \theta \rightarrow$ $U_{k}(\theta, x)$ is bounded and measurable with respect to $\mathcal{S}$. Players maximize the expected discounted sum of payoffs, and $\delta \in(0,1)$ is the discount factor.

In this game, denoted by $\Gamma^{\mathrm{en}}$, the strategic link across periods comes solely from the continuing nature of the policies. To characterize the impact of the endogenous status quo on the equilibrium behavior and outcomes, we will compare $\Gamma^{\mathrm{en}}$ to the game $\Gamma^{\mathrm{ex}}$, which differs from $\Gamma^{\mathrm{en}}$ only in that in every period $t$, the status quo is exogenously fixed at some $q(t) \in X$, irrespective of players' past actions.

As is customary in dynamic voting games with an infinite horizon, we look for Markov perfect equilibria in stage-undominated strategies as defined in Baron and Kalai (1993). ${ }^{3}$ A Markov strategy for player $k$ in $\Gamma^{\text {ex }}$ or $\Gamma^{\mathrm{en}}$, denoted by $\sigma_{k}$, maps in each period $t$ the current state $\theta(t)$ and status quo $q(t)$ into a probability distribution over votes. Stage undomination amounts to assuming that in each period, each player votes for the alternative that gives her the greater continuation payoff. It rules out pathological equilibria such as both players always voting for the status quo. Throughout the paper, the term equilibrium refers to this equilibrium concept unless stated otherwise. We derive all our results without placing any restrictions on how players vote when indifferent. However, when explaining the intuition in the text, we adopt the convention that they vote for $R$.

\footnotetext{
3 Stage undominated Markov perfect equilibria, or variants thereof, are used in almost all the infinite horizon models cited in this paper. Baron and Ferejohn (1989) show that requiring only subgame perfection leads to a folk theorem. As shown in Baron and Kalai (1993), stage undominated Markov perfect equilibria have a focal point property that de rives from their simplicity. Markov perfection in the legislative sphere can be justified on the grounds that the game is played by a sequence of legislators who are never certain to be reelected. In such cases, the institutional memory required for more sophisticated nonsta tionary equilibria involving infinitely nested punishment strategies may be inappropriate. See Bhaskar, Mailath, and Morris (2013) for a formalization of this argument and Krehbiel (1991) for a critical discussion of the prevalence of folk theorem like cooperation among legislators.
} 


\section{B. Additional Assumptions and Notation}

Definition 1. A payoff function $U: \Theta \times X \rightarrow \mathbb{R}$ is more leftist than another payoff function $U^{\prime}$ (or, equivalently, $U^{\prime}$ is more rightist than $U$ ) if for all $\theta \in \Theta$,

$$
U(\theta, R) \quad U(\theta, L) \leq U^{\prime}(\theta, R) \quad U^{\prime}(\theta, L) .
$$

Throughout, we assume that $U_{r}$ is more rightist than $U_{l}$, and we occasionally refer to players $r$ and $l$ as the rightist and the leftist players, respectively. This assumption has a natural interpretation in political economy applications: players can be unambiguously ranked on the ideological spectrum. Note, however, that it imposes no restrictions on the preference distribution of a single player nor on the severity of the conflict of interest between players: both players might prefer the same alternative for an arbitrarily large subset of $\Theta$.

For all $\theta \in \Theta$, let $U_{k}(\theta) \triangleq U_{k}(\theta, R) \quad U_{k}(\theta, L)$ be the relative period payoff for player $k$ from alternative $R$ as compared to $L$ in a period in which the state is $\theta$. We call $U_{k}(\theta)$ player $k$ 's current preference, as the sign of $U_{k}(\theta)$ determines her preference for the current period without taking into account the consequences of today's bargaining outcome on future periods. Throughout the paper, for any pair of functions or scalars $f_{l}, f_{r}$, we denote $f=\left(f_{l}, f_{r}\right)$. For example, we denote $U=\left(U_{l}, U_{r}\right)$.

To illustrate our results, sometimes we will use the following family of payoff profiles in which each player $k$ has single-peaked preferences with a peak equal to the state $\theta$ plus a player-specific shift $b_{k}$. This shift can be interpreted as her ideological bias.

Definition 2. When $\Theta \subseteq \mathbb{R}$ and $X \subset \mathbb{R}$, a profile of payoff functions $U$ is quadratic if for all $k \in\{l, r\}, \theta \in \Theta$, and $x \in X, U_{k}(\theta, x)=\left[\begin{array}{ll}x & \left(\theta+b_{k}\right)\end{array}\right]^{2}$ for some $b_{l}, b_{r} \in \mathbb{R}$ such that $b_{l}<b_{r}$.

A few comments about the model are in order. First, we assume that in each period the outcome is decided by a simultaneous vote, but with two alternatives and two players, most within-period bargaining procedures are equivalent. ${ }^{4}$

Second, to assess the role of the endogenous status quo, we compare $\Gamma^{\mathrm{en}}$ to the game with the exogenous status quo protocol $\Gamma^{\mathrm{ex}}$. The exogenous status quo protocol is the simplest dynamic bargaining protocol that severs the link between today's agreement and tomorrow's status quo. Hence, this benchmark protocol allows us to show in a transparent way that it is the continuing nature of policies that leads to polarization.

Finally, in this model, today's bargaining outcome affects the future only via tomorrow's status quo. In a more general model, it could also af-

\footnotetext{
${ }^{4}$ For instance, using standard equilibrium concepts, equilibrium outcomes are the same when players vote simultaneously or sequentially, when they make take it or leave it offers, or when they make several alternating offers in each bargaining period.
} 
fect tomorrow's states of nature. We abstract from this dependence to isolate the dynamic linkage of the endogenous status quo in a transparent way.

\section{Equilibrium Analysis}

As a benchmark, we first look at the game with an exogenous status quo $\Gamma^{\mathrm{ex}}$. Consider player $k$ in period $t$. Since the bargaining outcome implemented in $t$ has no impact on the subgame starting in the next period $t+$ 1 , player $k$ votes for $R$ if and only if

$$
U_{k}(\theta(t)) \geq 0 .
$$

Hence, $\Gamma^{\mathrm{ex}}$ has a unique equilibrium in which players simply vote for the best alternative according to their current preferences.

Consider now the same situation in the game with an endogenous status quo $\Gamma^{\mathrm{en}}$. The alternative $x$ implemented in $t$ affects player $k$ 's payoff in $t$, and since $x$ becomes the status quo in $t+1$, it also affects the subgame that starts in $t+1$. Let $W_{k}^{\sigma}(\theta, x)$ be the expected value of that continuation game, conditional on $\theta(t)=\theta$ and on the strategy profile $\sigma$ being played after $t$. Let $W_{k}^{\sigma}(\theta) \doteq W_{k}^{\sigma}(\theta, R) \quad W_{k}^{\sigma}(\theta, L)$. One can interpret $W_{k}^{\sigma}$ as the relative expected gain for player $k$ from having $R$ instead of $L$ as the next period's status quo. With a slight abuse of notation, we call $W_{k}^{\sigma}$ the continuation value of player $k$.

Stage undomination requires that in each period, players vote as if they were pivotal. Hence, in $\Gamma^{\mathrm{en}}$, player $k$ votes for $R$ in $t$ if and only if

$$
U_{k}(\theta(t))+\delta W_{k}^{\sigma}(\theta(t)) \geq 0 .
$$

Comparing (1) and (2), one can see that the effect of the endogenous status quo on equilibrium behavior is completely captured by $W_{k}^{\sigma}$. If $W_{k}^{\sigma}(\theta)$ is positive (negative) for all $\theta \in \Theta$, then in $\Gamma^{\text {en }}$, player $k$ votes for $R$ in a larger (smaller) set of states than in $\Gamma^{\mathrm{ex}}$.

The first proposition characterizes the effect of the endogenous status quo on equilibrium behavior.

Proposition 1. In any equilibrium, for all $\theta \in \Theta, W_{l}^{\sigma}(\theta) \leq 0 \leq W_{r}^{\sigma}(\theta)$. Hence, in $\Gamma^{\text {en }}$, player $l$ votes for $L$ and player $r$ votes for $R$ for a larger set of states (in the inclusion sense) than in $\Gamma^{\mathrm{ex}}$.

Proof. All proofs are in the Appendix.

The intuition for proposition 1 is as follows. The next period's status quo affects the next period's bargaining outcome only if players disagree in that period, in which case the status quo stays in place. Under our assumption that $U_{r}$ is more rightist than $U_{l}$, when players disagree, $r$ prefers $R$ while $l$ prefers $L$. Hence, player $r$ prefers $R$ as the future status quo while player $l$ prefers $L$ as the future status quo, which explains $W_{l}^{\sigma}(\theta) \leq$ 
$0 \leq W_{r}^{\sigma}(\theta)$. Therefore, player $l$ is willing to sacrifice some of her payoff in the current period by distorting her voting behavior in favor of $L$ in order to secure $L$ as the next period's status quo. Similarly, player $r$ is willing to sacrifice some of her current payoff to secure $R$ as the next period's status quo.

Since $U_{r}$ is more rightist than $U_{l}$, from (1) we see that already under an exogenous status quo, player $r$ votes for $R$ in a larger (in the inclusion sense) set of states than player $l$. Hence, proposition 1 implies that the endogenous status quo has a polarizing effect: it exacerbates the conflict of interest between the leftist and rightist players. In what follows, we refer to this equilibrium behavior as strategic polarization.

Since, under the endogenous status quo, player $l$ votes for $L$ and player $r$ votes for $R$ for a larger set of states than under the exogenous status quo, the endogenous status quo leads to more status quo inertia.

Corollary 1. In any equilibrium of $\Gamma^{\mathrm{en}}$, the set of states in which the status quo stays in place is greater in the inclusion sense than in the equilibrium of $\Gamma^{\mathrm{ex}}$.

An important implication of corollary 1 is that the equilibrium outcomes may be Pareto inefficient even in the static sense. A sequence of policies $(x(t))_{t \in \mathbb{N}}$ is statically efficient if in each period $t^{\prime} \in \mathbb{N}$, there is no other policy $y$ that is strictly preferred by both players to $x\left(t^{\prime}\right)$, keeping the policies in the other periods unchanged. Under the endogenous status quo, there may be states in which both players would benefit from changing the policy without changing the status quo for the next period; but since no player can commit to such a temporary change, one player prefers to stay at the current status quo.

It is instructive to note that the magnitude of the strategic polarization identified in proposition 1 and corollary 1 is the result of a vicious cycle in which players' behaviors feed on themselves. To see this, observe that as player $l$ distorts her voting behavior in favor of $L$, players become less likely to reach an agreement in each period. This increase in the probability of future disagreement in turn makes player $r$ more willing to defend her preferred status quo $R$ and thus makes her distort her behavior in favor of $R$ even further.

Because of this strategic complementarity, there can be multiple equilibria that differ in their degree of strategic polarization. Since a more polarized behavior from a given player is detrimental to the other player, these equilibria can be Pareto ranked. We will say that for any two strategy profiles $\sigma$ and $\sigma^{\prime}, \sigma^{\prime}$ is more polarized than $\sigma$ if for all $\theta \in \Theta, W_{l}^{\sigma^{\prime}}(\theta) \leq$ $W_{l}^{\sigma}(\theta) \leq 0 \leq W_{r}^{\sigma}(\theta) \leq W_{r}^{\sigma^{\prime}}(\theta)$.

Proposition 2. There exists a least (most) polarized equilibrium, and this equilibrium is Pareto best (worst) among all equilibria.

We conclude this section with three comments. First, note that the only assumption we impose on players' payoff is that $U_{l}$ is more leftist than $U_{r}$. 
Hence, the direction in which the endogenous status quo biases players' behavior depends only on players' relative ideological positions. A player with a given $U_{k}$ is biased in favor of $L$ if she plays against a more rightist opponent, but she becomes biased in favor of $R$ if she plays against a more leftist opponent. The intuition behind this result is that since the status quo matters only when players disagree, players' preferences over the next status quo are given by their policy preferences conditional on disagreement, which depend only on players' relative ideology.

Second, we have established so far that if the endogenous status quo protocol distorts players' behavior relative to the exogenous status quo protocol, then these distortions increase disagreement and create status quo inertia. We have not established, however, that these distortions are strict, that is, that the equilibrium of $\Gamma^{\mathrm{ex}}$ is not an equilibrium of $\Gamma^{\mathrm{en}}$. However, for the equilibrium of $\Gamma^{\mathrm{ex}}$ to be also an equilibrium of $\Gamma^{\mathrm{en}}$, the process $\{\theta(t): t \geq 0\}$ must satisfy quite restrictive conditions. To see this, consider a sequence of states in which both players' current preferences favor $R$, but $l$ 's preferences for $R$ are vanishingly weak. Then in $\Gamma^{\mathrm{ex}}$, both players vote for $R$ in all such states while in $\Gamma^{\text {en }}$, if the probability that players' preferences disagree in the next period is not vanishingly small in the limit, $l$ will find it profitable to vote for $L$ instead of $R$. In Section III.E, we show that this is the case, for example, when payoffs are quadratic (see definition 2) and the state is independently and identically distributed (i.i.d.) over time.

Third, in this simple model, the inefficiency generated by the endogenous status quo arises only if preferences evolve over time. To see this, observe that if players' preferences were fixed, then in $\Gamma^{\mathrm{en}}$, each player would vote according to her current preferences as in $\Gamma^{\mathrm{ex}}$. The status quo could be replaced only in the first period, and the constant bargaining outcome would be trivially Pareto optimal. Hence, it is the combination of the endogenous status quo and the evolving environment that generates polarization and inefficient status quo inertia.

\section{The Determinants of Strategic Polarization and Gridlock Effect}

In this section, we investigate the main drivers of strategic polarization. Since strategic polarization is driven by the anticipation of future disagreement, it should be affected by the degree of players' conflict of interest and by their patience. Players whose current preferences are more likely to disagree, and who care more about the future, should be more willing to sacrifice today's payoff to obtain their preferred status quo for tomorrow's negotiations. The next proposition formalizes this intuition.

Proposition 3. Let $\left(U_{l}, U_{r}\right)$ and $\left(U_{l}^{\prime}, U_{r}^{\prime}\right)$ be two profiles of payoff functions such that $U_{l}^{\prime}$ is more leftist than $U_{l}$ and $U_{r}^{\prime}$ is more rightist than $U_{r}$ (in the sense of definition 1), and let $\delta$ and $\delta^{\prime}$ be two discount factors 
such that $\delta^{\prime} \geq \delta$. If $\sigma$ and $\sigma^{\prime}$ denote the Pareto best (worst) equilibria for the parameters $U_{l}, U_{r}$, and $\delta$ and for $U_{l}^{\prime}, U_{r}^{\prime}$, and $\delta^{\prime}$, respectively, then $\sigma^{\prime}$ is more polarized than $\sigma$.

Since strategic polarization leads to status quo inertia, proposition $3 \mathrm{im}$ plies that more patient and more ideologically polarized players are less likely to agree on Pareto-improving policies after a shock to the environment has made the status quo suboptimal.

The next proposition shows that the impact of the endogenous status quo on equilibrium behavior can be quite dramatic: under some conditions, there exists a gridlock equilibrium in which players never reach an agreement, and hence the bargaining outcome is totally unresponsive to the evolution of the environment.

Proposition 4. Let $\sigma^{g}$ be the strategy profile in which player $l$ always votes for $L$ and player $r$ always votes for $R$. Then $\sigma^{g}$ is an equilibrium if and only if for any $\theta \in \Theta$,

$$
E_{\theta(0)} \theta\left[\sum_{t=0}^{\infty} \delta^{t} U_{l}(\theta(t))\right] \leq 0 \leq E_{\theta(0)} \theta\left[\sum_{t=0}^{\infty} \delta^{t} U_{r}(\theta(t))\right] .
$$

Condition (3) requires that for any initial state, player $r$ 's ( $l$ 's) expected payoff from implementing $R$ in all future periods is greater (smaller) than her expected payoff from implementing $L$ in all future periods.

Note that whenever $\sigma^{g}$ is an equilibrium, it is necessarily most polarized, and so from proposition 2, it is also Pareto worst. Hence, proposition 3 implies that if gridlock is an equilibrium for some environment, then it remains an equilibrium when players become more patient or more ideologically polarized. The following corollary, however, demonstrates that the degree of ideological polarization needed for gridlock does not have to be large.

Corollary 2. Suppose that the process $\{\theta(t): t \geq 0\}$ is uniformly ergodic and that

$$
E\left[U_{l}(\tilde{\theta})\right]<0<E\left[U_{r}(\tilde{\theta})\right],
$$

where $\tilde{\theta}$ is a random variable distributed according to the stationary distribution of $\{\theta(t): t \geq 0\} .{ }^{5}$ Then there exists $\delta<1$ such that for all $\delta \geq \delta$, $\sigma^{g}$ is an equilibrium. ${ }^{6}$

\footnotetext{
${ }^{5}$ A stationary Markov chain with transition function $f$ is uniformly ergodic if, as $n \rightarrow \infty$, $f^{n}\left(\mu^{0}\right)$ converges to its unique stationary distribution uniformly over all initial distributions $\mu^{0}$ (see Meyn and Tweedie 1993, chap. 16). This condition is satisfied, for instance, when the Markov process is i.i.d. When $\Theta$ is finite, a (generic) sufficient condition for uniform ergodicity is that the transition matrix has all its entries strictly positive. Similar suffi cient conditions can be derived when $\Theta$ is compact (see, e.g., Meyn and Tweedie's theo rem 16.2.5).

${ }^{6}$ Note that the order of the qualifiers matters. There may not exist $\bar{\delta}<1$ such that, for all $\delta \geq \bar{\delta}$, gridlock is an equilibrium regardless of how close $E\left[U_{l}(\theta)\right]$ and $E\left[U_{r}(\theta)\right]$ are to zero.
} 
Corollary 2 implies that as long as (4) is satisfied, even players with arbitrarily similar preferences (i.e., $\left|E\left[U_{l}(\tilde{\theta})\right] \quad E\left[U_{r}(\tilde{\theta})\right]\right|$ arbitrarily small) may disagree forever if they are sufficiently patient. Note that condition (4) requires that players' payoffs are not identical, but it includes some environments in which players' current preferences always favor the same alternative. For example, consider an i.i.d. process with two equally likely states $\theta_{R}$ and $\theta_{L}$ such that $U\left(\theta_{R}\right)=(1,2)$ and $U\left(\theta_{L}\right)=(2,1)$. In this environment, for both players, $R$ is best in state $\theta_{R}$ and $L$ is best in state $\theta_{L}$; but for $\delta>2 / 3$, condition (3) is satisfied, so gridlock is an equilibrium. ${ }^{7}$

Clearly, in the last example, players always agreeing on the Paretoefficient policy is an equilibrium as well. The next section, however, shows an example of an environment in which gridlock is the unique equilibrium for sufficiently patient players.

\section{E. An Example with Quadratic Preferences and I.I.D. Shocks}

In this section, we illustrate our results in the following simple environment. The profile of payoff functions $U$ is quadratic (see definition 2), $R=1, L=1$, and the state is drawn in an i.i.d. fashion across periods.

For the quadratic payoff specification, the current preferences are given by $U_{k}(\theta)=4\left(\theta+b_{k}\right)$. Substituting this expression in (1) and in (2), we obtain that under an exogenous status quo, each player $k$ votes for $R$ if and only if $\theta \geq b_{k}$, while under an endogenous status quo, player $k$ votes for $R$ if and only if $\theta \geq b_{k} \quad \delta W_{k}(\theta) / 4$. Since today's state does not affect future states, $W_{k}(\theta)$ is independent of $\theta$. Therefore, in the equilibria of $\Gamma^{\mathrm{ex}}$ and $\Gamma^{\mathrm{en}}$, each player $k$ plays a cutoff strategy with the cutoff $b_{k}$ and $b_{k} \quad \delta W_{k} / 4$, respectively.

Denote $p_{k}=\delta W_{k} / 4$. The above implies that in the game with an endogenous status quo, players behave as if the status quo were exogenous but their ideological biases were $b+p$ instead of $b$. From proposition 1, in any equilibrium of $\Gamma^{\mathrm{en}}, p_{l} \leq 0 \leq p_{r}$. Hence, since $b_{l}<b_{r}$, the strategic polarization as captured by $p$ moves players farther apart on the ideological spectrum.

It is instructive to see how $W_{k}$, and thus $p_{k}$, are determined. Recall that $W_{k}$ measures the expected gain for player $k$ in period $t$ from having $R$ instead of $L$ as the status quo in $t+1$. The status quo in $t+1$ matters only if players' votes disagree in that period, that is, when $\theta(t+1) \in\left[b_{r}\right.$ $\left.\delta W_{r} / 4, b_{l} \delta W_{l} / 4\right)$, in which case the status quo stays in place. For any such $\theta(t+1)$, the continuation payoff from implementing $R$ relative

\footnotetext{
${ }^{7}$ We thank an anonymous referee for suggesting this example to us.
} 


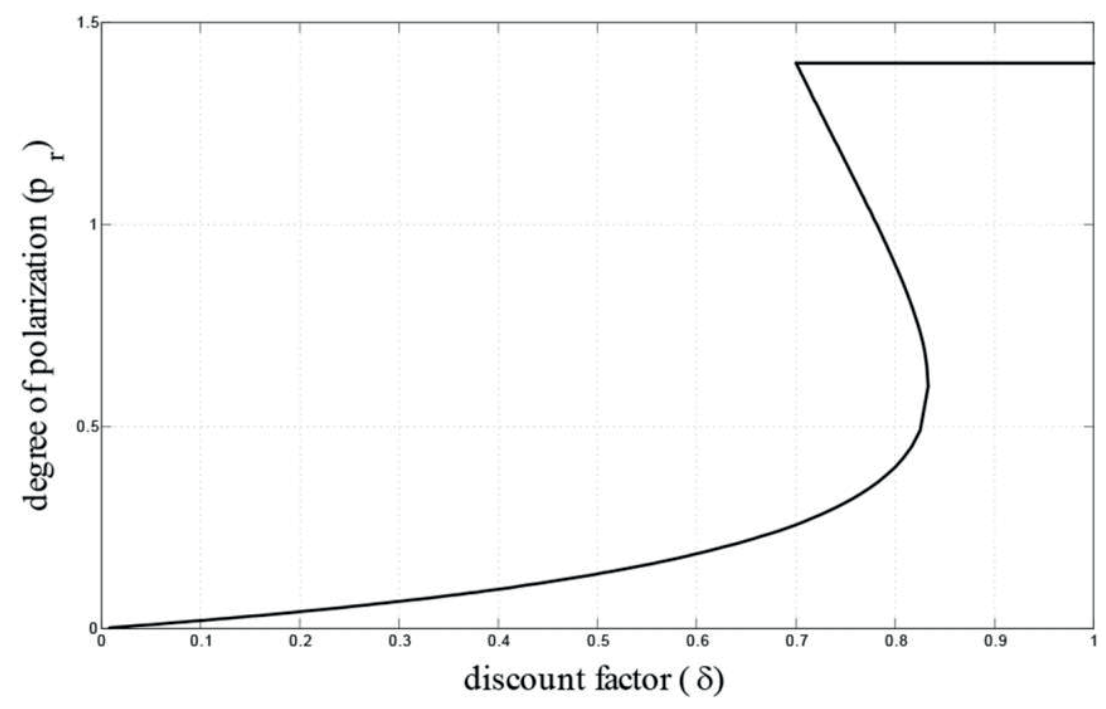

FIG. 1. Equilibrium $p_{r}$ as a function of $\delta$

to $L$ is $U_{k}(\theta(t+1))+\delta W_{k}$. Hence, in equilibrium, $W_{k}$ must satisfy

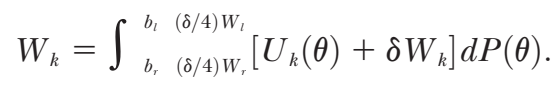

Figure 1 depicts the equilibrium $p_{r}$ in the symmetric case in which $b_{r}=$ $b_{l}=0.6$ and $\theta(t)$ is drawn uniformly on $\Theta=[2,2]$ (by symmetry, $p_{l}=$ $p_{r}$ ). For $\delta<0.7$, the equilibrium is unique and the corresponding $p_{r}$ strictly increases in $\delta$. When $\delta \geq 0.7$, condition (3) is satisfied, so the gridlock equilibrium exists (which corresponds to $\left.p_{r}=1.4\right)$. For $\delta \in(0.7$, 0.83 ), there are three equilibria, and for $\delta \geq 0.83$, only the gridlock equilibrium remains. In that case, under an exogenous status quo, players reach an agreement with probability .7 (when $b_{r} \leq \theta<b_{l}$ ), but they never do so under an endogenous status quo.

\section{The N-Alternative Model}

The two-alternative model allows us to isolate in a transparent way the polarizing effect of the endogenous status quo. In this section, we argue that under reasonable conditions, this effect is robust to the number of alternatives.

\section{A. Polarization and Moderation: Intuition}

With an arbitrary number of alternatives, it is still true that the status quo matters only when players disagree, so players' equilibrium behavior still 
depends on which policies they prefer when they disagree. However, players' preferences conditional on disagreement are harder to determine, because there is more than one way to disagree. Moreover, the consequences of some types of disagreement depend on the within-period bargaining procedure (i.e., the procedure that is used to select the bargaining outcome in each period).

First, as in the two-alternative model, players might disagree about which policies are better than the status quo. For example, in the context of legislative bargaining over tax policies, the conservatives may want to decrease tax rates while the liberals may want to increase them. In such disagreement states, the status quo stays in place irrespective of the withinperiod bargaining procedure. Hence, a higher (lower) status quo results in a higher (lower) tax rate. When the status quo is endogenous, the anticipation of such disagreement biases the conservatives in favor of lower and the liberals in favor of higher taxes. This is the same polarizing effect as in the case of two alternatives.

However, players may also agree that a certain subset of policies is better than the status quo but disagree about the ranking of these alternatives. In such disagreements, the bargaining outcome might not vary monotonically with the status quo. To see this, consider again the case of legislators bargaining over taxes, and suppose that a shift in investors' sentiment over the sustainability of the deficit makes increasing taxes desirable. In that case, all legislators may want to increase taxes, but the liberals will want to increase them more. If the status quo tax rates are excessively low given the needs and if the liberals can make a take-it-or-leave-it offer, they will propose a greater tax increase than what is optimal for the conservatives, and the conservatives will have no choice but to accept it. In this case, a slightly higher status quo would have constrained the bargaining power of the liberals and resulted in a lower tax rate. When the status quo is endogenous, the anticipation of this scenario biases the conservatives against low taxes. Thus, the endogenous status quo can also have a moderating effect.

The two effects have opposite implications in terms of the dynamics of the bargaining outcome. The polarizing effect induces players to disagree more often than their current preferences do and thus leads to status quo inertia. In contrast, the moderating effect biases players' behavior toward alternatives that are typically favored by the opponent. Therefore, it may induce a player to agree on a policy change even if her current preferences do not favor it; that is, a new policy may be approved even if it is $\mathrm{Pa}$ reto dominated by the status quo. This means that moderation can lead to status quo instability. Hence, whether the endogenous status quo induces policy inertia as in the two-alternative model depends on which of these two effects dominates. In Sections IV.B and IV.C, however, we identify two arguably relevant environments in which the polarizing effect dominates and status quo inertia prevails. 
In Section IV.B, we assume that within each period, the bargaining outcome is selected through the consensus procedure (Gradstein 1999; Dal Bó 2006; Riboni and Ruge-Murcia 2010): players revise the status quo through a series of incremental changes. We show that under this withinperiod bargaining procedure, the polarizing effect dominates. To understand this result, note that from the above discussion, the polarizing effect occurs irrespective of the within-period bargaining procedure. For the moderating effect to occur, however, the bargaining procedure must favor more drastic policy changes whenever players agree on the direction of the change but not its magnitude. By virtue of being incremental, the consensus procedure favors less drastic policy changes, thereby eliminating the moderating effect.

There are reasons to believe that the consensus procedure is a reasonable description of the negotiations that occur in some decision-making bodies. First, since the seminal work of Lindblom (1959) and Wildavsky (1992), a large body of literature on policy processes has found that public decision making in pluralist societies tends to follow an incremental approach that is in line with the consensus procedure. Second, Riboni and Ruge-Murcia (2010) have found that in the case of monetary policy committees, the consensus procedure delivers outcomes that are consistent with empirical evidence.

In Section IV.C, we allow for a general class of within-period bargaining procedures but consider instead an environment in which preferences evolve smoothly over time and players are given the opportunity to revise the status quo arbitrarily frequently. We show that in such environments, status quo inertia prevails in equilibrium. To understand why, note that from the above discussion, to generate the type of disagreement that leads to moderation, the preferences must change drastically from one period to the next: players must agree on low taxes at some point in time, but both must prefer to increase them substantially when the next chance to renegotiate arises. Such events do not occur when players can revise the policy agreement as soon as the environment prompts it. We believe that the latter assumption is empirically relevant because in actual bargaining situations, bargaining parties are rarely constrained on when they can revise the status quo. ${ }^{8}$

\footnotetext{
${ }^{8}$ Suppose, for instance, that legislators cut taxes after an economic contraction, but quickly thereafter, the markets lose faith in the ability of the government to repay its debts. In that scenario, the legislators are unlikely to wait for the next ordinary legislative session to start negotiating over how to react to the new situation. Similarly, since 1981 the Federal Open Market Committee has held eight regularly scheduled meetings each year at inter vals of 58 weeks. If circumstances require, however, members may be called on to partic ipate in a special meeting. For example, during the financial crisis the committee met 11 times in 2007 and 14 in 2008 (source: http://www.federalreserve.gov/monetarypolicy /fomchistorical2008.htm).
} 


\section{B. Bargaining with the Consensus Procedure}

The set of alternatives $X=\left\{x_{1}, x_{2}, \ldots, x_{N}\right\}$ is a subset of $\mathbb{R}$, with $x_{1}<\cdots<x_{N}$. As in the two-alternative model, we assume that the profile of payoff functions $\left(U_{k}(\theta, x)\right)_{k \in\{l, r\}, \theta \in \Theta, x \in X}$ is bounded and that player $r$ is more rightist than player $l$ in the sense that naturally extends definition 1: For all $\theta \in \Theta$ and all $x, y \in X$ such that $x<y, U_{l}(\theta, y) \quad U_{l}(\theta, x) \leq U_{r}(\theta, y) \quad U_{r}(\theta, x)$.

The game differs from the one analyzed in Section III only in that in each period, the bargaining outcome is decided using the consensus procedure. That is, each period is divided into multiple stages. In the first stage, the proposer $p r \in\{l, r\}$ (whose possibly random identity can depend on the state $\theta$ ) can play left or right. If she plays right (left), then in the subsequent stages of that period, players vote via the unanimity rule on how far to the right (left) they should incrementally move the status quo. Formally, if the status quo is $x_{n}$, players vote on whether to move to $x_{n+1}$ $\left(\begin{array}{ll}x_{n} & 1\end{array}\right)$. If one of them votes no, the game stops. If both vote yes, $x_{n+1}$ $\left(\begin{array}{ll}x_{n} & 1\end{array}\right)$ becomes the temporary agreement for the next stage. In the next stage, players vote on whether to move to $x_{n+2}\left(x_{n} 2\right)$, and so on. Bargaining continues until one player plays no or until the temporary agreement is $x_{N}$ $\left(x_{1}\right)$. The last temporary agreement $x$ becomes the bargaining outcome for that period, players obtain payoffs $\left(U_{k}(\theta, x)\right)_{k \in\{l, r\}}$, and the game moves to the next period with $x$ as the new status quo. The discounting $\delta \in(0,1)$ occurs only between periods. We call this game $\Gamma_{c}^{\mathrm{en}}$. Let $\Gamma_{c}^{\mathrm{ex}}$ denote the game that differs from $\Gamma_{c}^{\mathrm{en}}$ in that the status quo in each period $t$ is exogenously fixed at some $q(t)$.

\section{Conditions for Strategic Polarization}

As in the two-alternative model, the effect of the endogenous status quo on equilibrium behavior is captured by the value function $q \rightarrow W_{k}^{\sigma}(\theta, q)$. If $W_{k}^{\sigma}(\theta, q)$ is increasing (decreasing) in $q$, player $k$ favors replacing $x_{n}$ with $x_{n+1}$ in more (fewer) states than under the exogenous status quo. Hence, we will say that an equilibrium with a strategy profile $\sigma$ is polarized if for all $\theta \in \Theta, W_{r}^{\sigma}(\theta, q)$ is weakly increasing in $q$ and $W_{l}^{\sigma}(\theta, q)$ is weakly decreasing in $q .^{9}$

Proposition 5. If $\Theta$ is finite, there exists an equilibrium of $\Gamma_{c}^{\mathrm{en}}$ that is polarized.

We cannot ascertain that all equilibria are polarized, because if the proposer is indifferent between choosing left and right for some status quos, she can impose a nonmonotonic mapping between the status quo and the

\footnotetext{
${ }^{9}$ Note that for $|X| \quad$ 2, if we set $x_{1} \quad L$ and $x_{2} \quad R$, this definition of strategic polariza tion is equivalent to the definition used in Sec. III.
} 
outcome. As explained in Section IV.A, this implies that the rightist (leftist) player may not necessarily prefer more rightist (leftist) status quos.

In assumption 1 below, we define a large class of environments in which such indifferences occur with negligible probability and show that in these environments, all equilibria are polarized. Assumption 1 requires that players' payoffs depend on a stochastic parameter $\theta_{1}(t)$ that is arbitrarily correlated across time and on a shock $\theta_{2}(t)$ that smoothly perturbs the payoffs (condition i) but does not affect the distribution of future payoffs (condition ii).

Assumption 1. In all periods $t$, the state can be decomposed into two coordinates $\theta(t)=\left(\theta_{1}(t), \theta_{2}(t)\right)$ such that

i. conditioning on $\theta_{1}(t)$ and $\theta_{1}(t+1),\left(U_{k}\left(\theta_{1}(t+1), \theta_{2}(t+1), x\right)\right)_{k \in\{l, r\}, x \in X}$ has an absolutely continuous distribution on $\mathbb{R}^{\{l, r\} \times X}$;

ii. conditioning on $\theta_{1}(t), \theta_{1}(t+1)$ and $\theta_{2}(t+1)$ are independent of $\theta_{2}(t)$.

Note that the payoff perturbation induced by $\theta_{2}(t)$ can have an arbitrarily small support. So the environment described in assumption 1 can be viewed as an infinitesimal perturbation of the environment in which the (arbitrary) Markov process $\left\{\theta_{1}(t): t \geq 0\right\}$ is the only payoff-relevant state. Alternatively, if $\left\{\theta_{1}(t): t \geq 0\right\}$ is deterministic, assumption 1 encompasses the case in which players' payoffs are independently distributed over time.

Proposition 6. If assumption 1 is satisfied, then all equilibria are polarized. Furthermore, if $\Theta_{1}$ is finite, an equilibrium exists.

\section{The Case of Quadratic Preferences and Random Walk}

Propositions 5 and 6 show that players' equilibrium behavior exhibits strategic polarization but are silent on how the set of alternatives $X$ affects the magnitude of polarization. In this section, we analyze a reasonable environment in which it is independent of $X$ and is exactly the same as in the twoalternative model.

The profile of payoff functions $U$ is quadratic (see definition 2), and the process $\{\theta(t): t \in \mathbb{N}\}$ is a random walk on $\Theta=\mathbb{R}$ : for all $t \in \mathbb{N}, \theta(t+1)=$ $\theta(t)+\nu(t)$, where the random variables $(\nu(t))_{t \in \mathbb{N}}$ are i.i.d. and integrable. ${ }^{10}$ We further assume that each $\nu(t)$ admits a probability density function $f$ that is single-peaked with a peak at zero.

\footnotetext{
${ }^{10}$ The careful reader will note that since $\Theta \quad \mathbb{R}$, quadratic $U$ violates the assumption that $U$ is bounded over $\Theta$. However, the results stated in this section are derived without this assumption.
} 
Let us first analyze the equilibrium behavior with an exogenous status quo. Once the direction is chosen, say right, whenever voting between a temporary status quo $x_{n}$ and a change to $x_{n+1}$, the unique stageundominated action for each player is to vote yes if and only if $x_{n+1}$ is closer to her peak than $x_{n}$. Given this behavior at the voting stages, a stageundominated action for the proposer is to propose the direction of her peak, and all stage-undominated actions are outcome equivalent.

Consider a period with state $\theta$ and status quo $x_{n}$. Since the payoffs are quadratic, the strategy profile described above moves the policy to the right if both players prefer $x_{n+1}$ to $x_{n}$, which happens when $\theta \geq\left(x_{n+1}+\right.$ $\left.x_{n}\right) / 2 \quad b_{l}$. Likewise, the policy moves to the left if both players prefer $x_{n}$ to $x_{n}$, which happens when $\theta<\left(x_{n}{ }_{1}+x_{n}\right) / 2 \quad b_{r} \cdot{ }^{11}$ Hence, the status quo $x_{n}$ stays in place if

$$
\theta \in\left[\frac{x_{n}+x_{n} 1}{2} \quad b_{r}, \frac{x_{n+1}+x_{n}}{2} \quad b_{l}\right) .
$$

Proposition 7. There exist $p_{l}<0$ and $p_{r}>0$ such that for all finite $X \subset \mathbb{R}$, the equilibrium of $\Gamma_{c}^{\text {en }}$ with players' biases $b+p$ is an equilibrium of the game $\Gamma_{c}^{\mathrm{en}}$ with players' biases $b$.

Proposition 7 states that with an endogenous status quo, there exists an equilibrium in which players behave as if the status quo were exogenous but their biases were $b+p$ instead of $b$. Since $b_{l}<b_{r}$ and $p_{l}<0<p_{r}$, the strategic polarization again moves players further apart on the ideological spectrum. Together with (5), proposition 7 implies that the status quo $x_{n}$ stays in place when

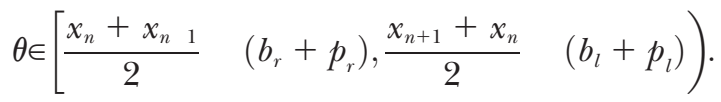

By comparing (5) and (6), we see that as in the two-alternative model, the endogenous status quo increases status quo inertia, more so the greater the polarizing effect $p$.

Note that proposition 7 states that $p_{l}$ and $p_{r}$ are independent of $X$. Hence, the endogenous status quo generates the same magnitude of polarization and status quo inertia independent of the number and similarity of the available alternatives. Moreover, since for $|X|=2$ the equilibria of $\Gamma_{c}^{\mathrm{en}}$ are outcome equivalent to the equilibria of $\Gamma^{\mathrm{en}}$ analyzed in Section III.C, proposition 7 implies that the polarizing effect is exactly as in the two-alternative model. Hence, consistent with the intuition laid out in Section IV.A, there is no moderating effect in this setting.

${ }^{11}$ Recall that in the text we adopt the convention that players vote for the more rightist alternative when indifferent. 


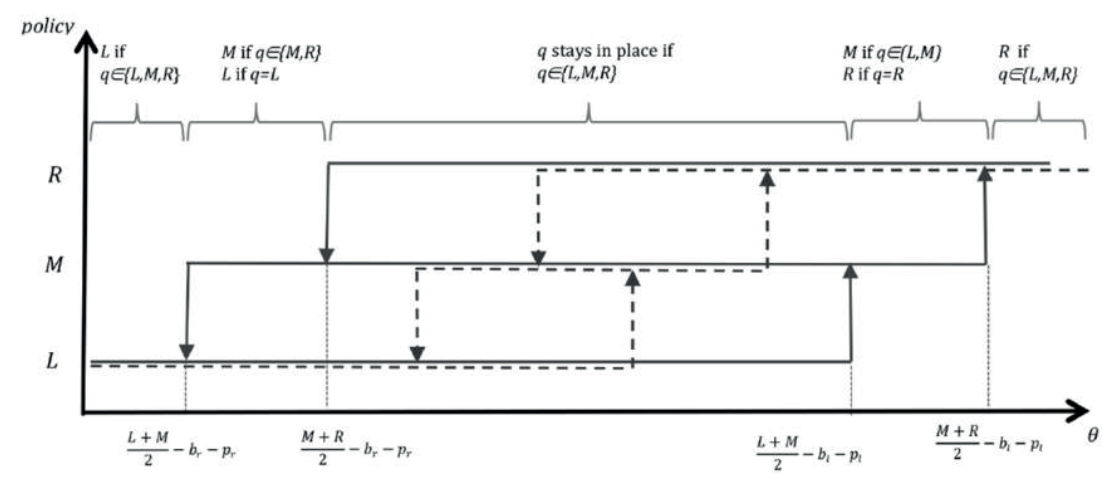

FIG. 2. The evolution of the policy

Figure 2 depicts the evolution of the equilibrium outcome in $\Gamma_{c}^{\mathrm{en}}(\{L$, $M, R\}, b)$ (solid lines) and $\Gamma_{c}^{\mathrm{ex}}(\{L, M, R\}, b)$ (dashed lines). The horizontal lines represent the set of states in which a given status quo stays in place, and the arrows represent the cutoff states at which the policy switches.

\section{Bargaining in a Continuously Changing Environment}

In this section, we generalize the model of Section IV.B in that we allow for a broad class of within-period bargaining procedures but assume that preferences evolve continuously and players can bargain arbitrarily frequently.

The set of alternatives $X$ is finite. Time is continuous, but players bargain only every $\Delta>0$ units of time. At each bargaining time $t \in\{0, \Delta$, $2 \Delta, \ldots\}$ with status quo $q$ and state $\theta$, players play a within-period bargaining procedure denoted by $\wp(\theta, q)$ whose outcome determines players' flow payoff for the period for $[t, t+\Delta)$ and becomes the status quo at $t+\Delta$. The state $\theta(t)$ follows a continuous-time stochastic Markov process on $\Theta$. The flow payoff of player $k \in\{l, r\}$ in state $\theta$ from alternative $x \in X$ is denoted by $u_{k}(\theta, x)$ and is uniformly bounded. Players maximize the expected discounted sum of flow payoffs, and $\rho>0$ is the discount factor. We denote this game by $\Gamma_{\wp}^{\mathrm{en}}(\Delta)$.

Before deriving the formal result, we use a simple example to illustrate how polarization, moderation, and status quo inertia depend on $\Delta$ and on $\wp$.

1. A Numerical Example with the Agenda-Setter Procedure

Suppose that $X=\{1,0,1\}$, the profile of flow payoffs is quadratic (see definition 2), and $\{\theta(t): t \in \mathbb{N}\}$ is a standard Brownian motion on $\Theta=$ $\mathbb{R}$. Conditional on $\theta(t), \theta(t+\Delta)$ is distributed according to $N(\theta(t), \sqrt{ } \Delta)$. 
If we set $U_{k}(\theta, x)=E_{\theta(0)}{ }_{\theta}\left[\int_{0}^{\Delta}\left[u_{k}(\theta(t), x)\right] \rho e^{\rho t} d t\right]$, then $U_{k}(\theta, x)$ is quadratic (modulo a constant). Hence, if $\wp=c$ denotes the consensus procedure, then the game $\Gamma_{c}^{\mathrm{en}}(\Delta)$ is equivalent to the game analyzed in Section IV.B with $\delta=e^{\rho \Delta}$ and $f$ being the density of the normal distribution with $\mu=$ 0 and $\sigma=\sqrt{ } \Delta$.

Consider now the agenda-setter procedure, denoted by $\wp=r$, in which $r$ makes a take-it-or-leave-it offer to player $l$ in each period. By analogy with the equilibrium of $\Gamma_{c}^{\mathrm{en}}(\Delta)$ characterized in proposition 7, we postulate the following cutoff equilibrium of $\Gamma_{r}^{\text {en }}(\Delta)$ : for each pair of consecutive alternatives, $x_{n}$ and $x_{n+1}$, there exists $p_{x_{n}, x_{n+1}}$ and $p_{x_{n+1}, x_{n}}$ such that when $q=x_{n}, r$ proposes a change to the right when $\theta \geq\left(x_{n}+x_{n+1}\right) / 2 \quad\left(b_{l}+p_{x_{n}, x_{n+1}}\right)$, and when $q=x_{n+1}, r$ proposes a change to the left when $\theta<\left(x_{n}+\right.$ $\left.x_{n+1}\right) / 2 \quad\left(b_{r}+p_{x_{n+1}, x_{n}}\right)$. All proposals of $r$ are accepted by $l{ }^{12}$ Note that this equilibrium of $\Gamma_{r}^{\mathrm{en}}(\Delta)$ exhibits less polarization than the equilibrium of $\Gamma_{c}^{\mathrm{en}}(\Delta)$ if $p_{x_{n}, x_{n+1}}>p_{l}$ and $p_{x_{n+1}, x_{n}}<p_{r}$, where $p_{l}$ and $p_{r}$ are players' degree of strategic polarization in the equilibrium of $\Gamma_{c}^{\mathrm{en}}(\Delta)$ characterized in proposition 7 .

The intuition laid out in Section IV.A suggests that under the procedure $\wp=r$, the moderating effect should bias player $l$ in favor of a moderate status quo $q=0$ relative to an extreme leftist one $q=1$ (and vice versa for player $r$ ), that is, $p_{1,0}>p_{l}$ and $p_{0,1}<p_{r}$. The reason is that when both players want to move the policy to the right but disagree by how much, a very leftist status quo $q=1$ allows the proposer $r$ to force $l$ to accept a drastic change of policy to $x=1$, while a moderate status quo $q=$ 0 does not. However, the moderating effect should vanish as $\Delta \rightarrow 0$; that is, $p_{1,0} \quad p_{l}$ and $p_{0,1} \quad p_{r}$ should tend to zero, because as players can revise the policy sufficiently frequently, the probability that they agree on 1 in some period but both prefer 1 in the next is negligible.

Figure 3 plots our numerical estimation of $\left(p_{l}, p_{r}\right)$ (solid) and $\left(p_{1,0}\right.$, $\left.p_{0,1}\right)($ dotted) as a function of $\Delta$ for $\rho=0.001$. As intuited, the moderating effect arises in $\Gamma_{r}^{\mathrm{en}}(\Delta)$ but vanishes as $\Delta \rightarrow 0 .{ }^{13}$ Interestingly, figure 3 also shows that under both the consensus and the agenda-setter procedures, players' degree of polarization increases as $\Delta \rightarrow 0$, so there is strict status quo inertia in the limit. ${ }^{14}$

12 This is only a partial description of the equilibrium but is sufficient for the point made in this example.

${ }^{13}$ For all parameters we used, we also obtain that there is no moderation effect between $q \quad 1$ and $q \quad 0$, i.e., $p_{0,1} \quad p_{l}$ and $p_{1,0} \quad p_{r}$. The intuition for this result is that with a rightist status quo, if both players want to move the policy to the left but disagree by how much, under $\wp \quad r$, the player who wants the more drastic change, i.e., $l$, never has any bargaining power.

${ }^{14}$ All simulation results are available from the authors on request. 


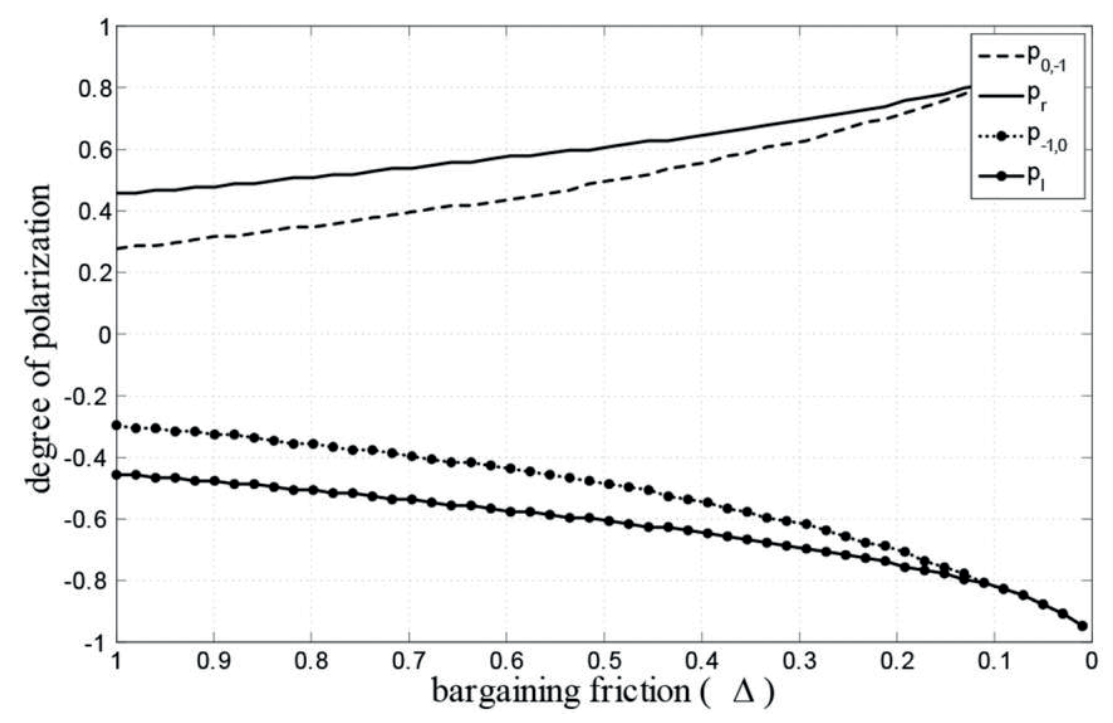

FIG. 3. Strategic polarization in $\Gamma_{c}^{\mathrm{en}}$ and $\Gamma_{r}^{\mathrm{en}}$

\section{General Result: Status Quo Inertia}

The only restriction we impose on $\wp$ is that either player can unilaterally block any change away from the status quo. Formally, for all $\theta \in \Theta$ and $q \in$ $X$, in any stage-undominated subgame perfect equilibrium of the stage game $\wp(\theta, q)$, with probability one, both players weakly prefer the equilibrium outcome to the status quo $q \cdot{ }^{15}$ To capture the requirement that preferences evolve smoothly over time, we assume that the state space $\Theta$ is a topological space such that for all $x \in X, u_{k}(\theta, x)$ is continuous in $\theta$, and the process $\{\theta(t): t \geq 0\}$ satisfies the following condition.

Assumption 2. For all $\theta \in \Theta$ and all neighborhoods $B$ of $\theta$, if $\hat{t}_{B}$ denotes the first exit time of $\theta(t)$ from $B$, then $\operatorname{Pr}_{\theta(0)}{ }_{\theta}\left(\hat{t}_{B} \leq t\right)=o(t)$ as $t \rightarrow 0 .{ }^{16}$

Let $\phi^{\sigma}(\theta, q ; x)$ denote the probability that $x$ is implemented in a bargaining period $t$ if $\theta(t)=\theta, q(t)=q$, and players play strategy profile $\sigma$

15 This assumption is satisfied, for instance, for any state dependent agenda setter pro cedure and for the voting procedure from Sec. III. The consensus procedure satisfies this restriction in any pure strategy equilibrium and in any mixed strategy equilibrium if as sumption 1 holds. Our conclusions also hold in the case in which the bargaining outcome within each period is determined by some cooperative bargaining solution such as the Nash bargaining solution, where players' payoffs are their continuation value from imple menting a given alternative.

${ }_{16}$ Assumption 2 is satisfied for all standard continuous Markov processes such as the Brownian motion used in Sec. IV.C.1. See remark 1 in the online appendix. Note also that if $\{\theta(t): t \geq 0\}$ is deterministic, then assumption 2 is satisfied if and only if $\{\theta(t): t \geq 0\}$ is continuous. When $\Theta$ is a metric space, it is satisfied if $\{\theta(t): t \geq 0\}$ is Lipschitz continuous in $t$ with probability one. 
in $\Gamma_{\wp}^{\text {en }}(\Delta)$. Consider a sequence $\left(\Delta^{s}\right)_{s \in \mathbb{N}}$ such that $\Delta^{s} \rightarrow 0$ and a sequence of strategy profiles $\left(\sigma^{s}\right)_{s \in \mathbb{N}}$ such that for all $s \in \mathbb{N}, \sigma^{s}$ is an equilibrium of $\Gamma_{\wp}^{\mathrm{en}}\left(\Delta^{s}\right)$. This sequence is fixed throughout.

We are ready to state our result.

Proposition 8. Let $\theta \in \Theta$ and $q \in X$. Suppose that there exists $x \in X$, a neighborhood $B$ of $\theta$, and some $\varepsilon>0$ such that for $s$ sufficiently large, for all $\zeta \in B, \phi^{\sigma^{\prime}}(\zeta, q, x)>\varepsilon$ and $\phi^{\sigma^{s}}(\zeta, x, x)=1$. Then for all $k \in\{l, r\}, u_{k}(\theta, x) \geq$ $u_{k}(\theta, q)$.

Proposition 8 characterizes the equilibrium behavior as $\Delta \rightarrow 0$ only in the states $\theta$ in the neighborhood of which status quo $q$ is replaced with a nonvanishing probability by some bargaining outcome, which in turn is not replaced immediately unless the state leaves the neighborhood of $\theta$. However, in the equilibria characterized in Sections IV.B and IV.C.1, this is the case for all $q$ and almost all $\theta \cdot{ }^{17}$

Proposition 8 states that when the negotiating parties can revise the bargaining agreement sufficiently frequently, the status quo $q$ can be replaced by $x$ in a neighborhood of some $\theta$ only if $x$ Pareto improves on $q$ in terms of the current preferences at $\theta$. Note that with an exogenous status quo, the status quo $q$ is replaced exactly when an alternative $x$ Pareto improves on $q$ in terms of the current preferences. Hence, proposition 8 shows that if the endogenous status quo distorts the dynamics of the bargaining outcome relative to the exogenous status quo, this distortion can take the form only of status quo inertia.

\section{Applications to Legislative Bargaining}

Most laws and policies enacted by the US Congress are permanent: they remain in effect until a new legislative action is taken. ${ }^{18}$ As such, they fit

\footnotetext{
${ }^{17}$ To see this, note that these equilibria are in pure strategies and the set of states at which a given action is prescribed is an interval of $\Theta \mathbb{R}$. Hence, for any sequence $\left(\sigma^{s}\right)_{s \in \mathbb{N}}$ of such equilibria and any $q$, there exists a subsequence in which these intervals con verge. In any state $\theta$ that does not coincide with the limit of the boundaries of these inter vals, the actions and thus the bargaining outcome prescribed by $\sigma^{s}$ are locally constant around $\theta$ for $s$ sufficiently large. Moreover, in these equilibria, the bargaining outcome around $\theta$ is not replaced unless the state changes. In general, there may be equilibria $\sigma$ that prescribe $q$ to be replaced by some $y$ for which $\phi^{\sigma}(\zeta, y, y)<1$ but that is replaced in state $\zeta$ by some $x$ for which $\phi^{\sigma}(\zeta, x, x) \quad 1$ (which could happen in some equilibrium, for instance, if $\mathcal{G}$ is the agenda setter, and $x$ and $y$ give exactly the same continuation payoff to the pro poser in state $\zeta$ ). Proposition 8 still holds in such cases, but the proof is more intricate. We refer the interested reader to Dziuda and Loeper (2012).

${ }^{18}$ Our model features two players, but in Dziuda and Loeper (2015), we extend it to an arbitrary number of legislators and general voting rules. We show that if legislators' relative ideological positions are constant across time (McCarty, Poole, and Rosenthal 2006), the voting rule determines two pivotal players whose agreement, as in the current model, is necessary and sufficient for a policy change in any equilibrium. As in the pivotal politics literature (Krehbiel 1999; Brady and Volden 2006), the leftist (rightist) player in our model can be interpreted as the most liberal (conservative) policy maker among the veto players.
} 
the assumptions of our model. This is the case of mandatory spending policies, which include all entitlements and currently amount to 59 percent of total federal spending (Levit and Austin 2014), constitutional amendments, most statutes in the US code, the Senate's rules of proceedings, or international treaties. Likewise, changes to the tax code are permanent unless legislators decide to attach a sunset provision, which historically has been the exception rather than the norm. ${ }^{19}$

\section{A. Polarization and Gridlock}

The finding of our paper that in a changing environment, the continuing nature of policies leads to polarization and gridlock resonates with the casual observations of the proceedings in the US Congress. As a result of changing demographics, new social risks, increasing public debt, and legislative complexity, there is a general consensus that important continuing policies such as entitlements, immigration laws, or the tax code are in need of a serious overhaul. Yet legislators have repeatedly failed to act on them.

Since there is a lot of disagreement in the US Congress also on temporary legislation (see, e.g., Keystone XL Pipeline), the endogenous status quo is admittedly not the only source of polarization and legislative inertia. As shown in the pivotal politics literature (Krehbiel 1999; Brady and Volden 2006), supermajority requirements or veto points can create gridlock even for temporary legislation: as long as the status quo lies in between the ideal points of the two pivotal players - the "gridlock interval"any policy change will be vetoed. Our paper complements this literature by showing that in a dynamic setting, the continuing nature of policies makes these pivotal voters behave as if their ideal points were farther apart (see Secs. III.E and IV.B) and thus increases the size of the gridlock interval. Indeed, it may be telling that many controversial but temporary legislations such as the Bush tax cuts of 2001 and 2003, the Patriot Act of 2001, the Troubled Asset Relief Program plan, or Obama's fiscal stimulus in 2008 passed relatively swiftly, while the much needed reforms of the tax code or the entitlement system have not even reached the floor.

${ }^{19}$ See, e.g., Posner and Verneule $(2002,1672,1694,1701)$ on the permanent nature of statutes, the Senate's internal rules, or international treaties. As for tax legislation, prior to the Bush administration, sunsets applied mainly to relatively small provisions known as "tax extenders" (Gale and Orszag 2003). As Mooney (2004) puts it, "the use of sweeping sunset provisions in the tax code under the Bush Administration [referring to the sunset provi sions in the Economic Growth and Tax Relief Reconciliation Act of 2001 and the Jobs and Growth Tax Relief Reconciliation Act of 2003] represents a massive departure from previous tax policy." 
Our results (proposition 4 and corollary 2) show that strategic polarization and status quo inertia increase with legislators' discount rate. If legislators care about policies more when in office, their discount rate is linked to their reelection probability. In the case of the US Congress, the incumbency reelection rate has been very high (above 80 percent in the Senate and 90 percent in the House since the 1960s; see Friedman and Holden 2009, fig. 1), which suggests that the inertial effect of the endogenous status quo may be quite important. Our findings also suggest, counterintuitively, that political instability and term limits can be beneficial.

\section{B. Legislators' Ideology and Polarization}

Our paper adds to the discussion on legislators' ideology and polarization. Most of the literature measures legislators' ideal points on the leftright spectrum using Nominate scores derived from roll-call votes (Poole and Rosenthal 1997). The implicit premise of this empirical exercise is that legislators vote according to their true preferences. Our results imply that when voting on permanent policy changes, legislators may bias their voting behavior. As a result, Nominate scores may systematically overestimate legislators' ideological polarization.

The above observation resonates with the empirical findings in Bafumi and Herron (2010) that members of Congress are more extreme than the median voter of their respective constituency, where the former is measured by the voting record of the legislators and the latter by survey responses (see also Fiorina, Abrams, and Pope 2006; Fiorina and Abrams 2008; Krasa and Polborn 2014). The difference between legislators' and voters' polarization is usually interpreted as a failure of political representation. Our paper suggests an alternative interpretation: even if legislators' preferences coincide with that of their median constituent, the voting records of forward-looking legislators may exhibit more polarization than the preferences expressed by their constituents in nonbinding surveys.

The implication of our model that Nominate scores might be biased can be indirectly tested along the following lines. The voting behavior on permanent legislations of a given liberal (conservative) legislator is predicted to be more liberal (conservative) as her reelection probability increases and as the other legislators become more polarized. In contrast, these factors should not affect legislators' behavior if they vote on temporary legislations or if they vote myopically as the Nominate methodology implicitly assumes. Such an analysis may not be straightforward, as many bills contain both types of policies, and legislators have some discretion about which policy changes are temporary or permanent, which may create an endogeneity problem. It is nevertheless interesting to note that the steady increase in the share of mandatory spending in the federal budget since the 
1960s (Levit and Austin 2014) has been accompanied by a sharp increase in polarization as measured by Nominate scores (McCarty et al. 2006).

\section{Temporary Legislation and Sunset Provisions}

This paper shows that linking today's agreement and tomorrow's status quo generates a detrimental polarizing effect. In the context of legislative bargaining, there are two natural ways to sever this link: the exogenous status quo and sunset provisions. ${ }^{20}$

The exogenous status quo is arguably the most prevalent alternative to the endogenous status quo. For example, discretionary spending policies (e.g., federal wages, "pork barrel" projects) require annual appropriations and are thus legislated under a fixed default of no spending (Levit and Austin 2014). However, the determination of the exogenous default policy can be controversial in many policy domains. For instance, there is arguably no natural default for income taxation or social security benefits. Moreover, exogenous status quo is certain to be socially beneficial only if the default policy is set optimally in all states, but this may be hard to implement if the optimal default policy depends on the state and the latter is not verifiable.

A sunset provision is a clause that specifies duration after which a legislative act automatically expires. Although sunset provisions have received little attention from economists, they have long been advocated by political theorists and legal scholars to address statute proliferation and obsolescence and political entrenchment of regulations and agencies. ${ }^{21}$ Our results suggest a novel rationale for sunsets: by allowing the legislators to change the law without affecting the future status quo, sunsets induce them to evaluate policies solely on the basis of their adequacy to the current environment and hence eliminate the polarizing effect.

Legal scholars have pointed out that sunset provisions generate various inefficiencies, which may discourage legislators from using them (Posner and Vermeule 2002; Kysar 2011). It has also been observed that even though sunset policy changes do not formally affect the status quo,

\footnotetext{
${ }^{20}$ Automatic stabilizers can also affect the dynamic linkage of the endogenous status quo. An automatic stabilizer is a clause that triggers an automatic change in a continuing policy in response to a change in certain observable indicators (e.g., price levels, unem ployment rate). In a related paper, Bowen et al. (2014) show that the endogenous status quo can be an optimal rule when the status quo can be fully contingent on the state of na ture. In practice, however, verifiability may be an issue.

${ }^{21}$ Jefferson ([1789] 1958), Lowi (1969), and Calabresi (1982) famously supported the systematic use of sunset provision under various forms to avoid legislative obsolescence and entrenchment. Sunset provisions have also been argued to improve scrutiny over new policies with uncertain effects and improve legislative response to temporary circum stances and new risks. See Gersen (2007) and the references therein.
} 
they make it politically harder to revert to it (Kysar 2011). Hence, the availability and the use of sunsets may not completely eliminate the perverse dynamic incentives highlighted in this paper. We leave a formal analysis of the role of sunset provisions in dynamic negotiations for future research.

\section{Appendix}

\section{A. Proofs for the Two Alternative Model}

\section{Notation}

Let $m$ be a bound on $\left|U_{k}(\theta, x)\right|$ over all $k \in\{l, r\}, \theta \in \Theta$, and $x \in X$, and let $\mathcal{F}$ be the set of mappings from $\Theta$ into [ $\left.m /\left(\begin{array}{ll}1 & \delta\end{array}\right), m /\left(\begin{array}{ll}1 & \delta\end{array}\right)\right]$ that are measurable with respect to the sigma algebra $\mathcal{S}$ of $\Theta$. In what follows, $f \quad\left(f_{l}, f_{r}\right)$ denotes an arbi trary pair of such mappings, and $0^{\mathcal{F}}$ denotes the mapping that is equal to zero for all $\theta \in \Theta$. For all $T \subseteq \Theta$ and $f \in \mathcal{F}^{2}$, denote

$$
\begin{aligned}
& D^{\prime}(f) \stackrel{\circ}{=}\left\{\theta \in \Theta: f_{l}(\theta)<0 \text { and } f_{r}(\theta)>0\right\}, \\
& \left.D^{\prime \prime}(f) \stackrel{\circ}{=} \theta \in \Theta: f_{l}(\theta)>0 \text { and } f_{r}(\theta)<0\right\} \text {, }
\end{aligned}
$$

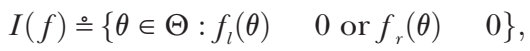

$$
\begin{aligned}
& D(T, f) \stackrel{\circ}{=} D^{\prime}(f) \cup D^{\prime \prime}(f) \cup(I(f) \cap T) .
\end{aligned}
$$

For all $T \subseteq \Theta$, we define the mappings $V$ and $\Omega^{T}$ as follows: for all $f \in \mathcal{F}^{2}, k \in\{l, r\}$, and $\theta \in \Theta$,

$$
\begin{gathered}
V_{k}\left(f_{k}, \theta\right) \stackrel{\circ}{=} U_{k}(\theta)+\delta f_{k}(\theta), \\
\Omega_{k}^{T}(f, \theta) \stackrel{\circ}{=} \int_{\zeta \in D(T, f)} f_{k}(\zeta) d P_{\theta}(\zeta),
\end{gathered}
$$

where $P_{\theta}$ denotes the probability distribution of $\theta(t+1)$, conditional on $\theta(t) \quad \theta$. When $f$ is equal to the continuation value $W^{\sigma}$ of some strategy profile $\sigma$ as defined in Section III.C, then $V_{k}\left(W_{k}^{\sigma}, \theta\right)$ is the relative gain in continuation payoff for player $k$ of implementing alternative $R$ instead of $L$ in period $t$ with state $\theta(t) \quad \theta$ in the game $\Gamma^{\mathrm{en}}$, conditional on $\sigma$ being played thereafter. Then $D^{\prime}\left(V\left(W^{\sigma}\right)\right)$ and $D^{\prime \prime}$ $\left(V\left(W^{\sigma}\right)\right)$ are the set of states in which players would strictly prefer to implement different alternatives given the continuation play $\sigma$, and $I\left(V\left(W^{\sigma}\right)\right)$ is the set of states in which some player is indifferent between the alternatives. Thus, the set $D(T, V(W))$ can be viewed as the set of states in which players disagree, with the convention that they disagree when they strictly prefer implementing different alternatives (i.e., when $\theta \in D^{\prime}\left(V\left(W^{\sigma}\right)\right) \cup D^{\prime \prime}\left(V\left(W^{\sigma}\right)\right)$ ) and when some player is in different and the state is in $T$. Hence, the set $T$ can be viewed as an arbitrary tie breaking rule that specifies how players vote when they are indifferent. Finally, $\Omega_{k}^{T}\left(V\left(W^{\sigma}\right), \theta\right)$ is the expectation of the relative gain for player $k$ of implementing alternative $R$ instead of $L$ in period $t+1$, conditional on $\theta(t) \quad \theta$, on continuation play $\sigma$, and on players disagreeing in $t+1$ in the above sense. 
We denote by $(\leq, \geq)$ the partial order on $\mathcal{F}^{2}$ defined as follows: for all $f, g \in \mathcal{F}^{2}$, $f(\leq, \geq) g$ if, for all $\theta \in \Theta, f_{l}(\theta) \leq g_{l}(\theta)$ and $f_{r}(\theta) \geq g_{r}(\theta)$. When $f$ and $g$ are continuation values of strategy profiles, this order corresponds to the polarization order on strategy profiles defined before proposition 2 .

\section{Preliminary Lemmas}

The following two lemmas show that a strategy profile is an equilibrium if and only if its continuation value is a fixed point of the map $f \rightarrow\left(\Omega^{T} \circ V\right)(f)$ and establish that this map is monotonic in the order $(\leq, \geq)$.

Lemma 1. If $\sigma$ is an equilibrium of $\Gamma^{\text {en }}$, then there exists $T \subset \Theta$ such that $W^{\sigma}$ is a fixed point of the map $f \rightarrow\left(\Omega^{T} \circ V\right)(f)$, and $\sigma$ prescribes players' votes to disagree in state $\theta$ if and only if $\theta \in D\left(T, V\left(W^{\sigma}\right)\right)$. Conversely, if $W$ is a fixed point of $f \rightarrow$ $\left(\Omega^{T} \circ V\right)(f)$ for some $T \subset \Theta$, then there exists an equilibrium $\sigma$ of $\Gamma^{\mathrm{en}}$ such that $W^{\sigma} \quad W$ and $\sigma$ prescribes players' votes to disagree in state $\theta$ if and only if $\theta \in$ $D(T, V(W))$.

Proof. Let $\sigma$ be an equilibrium and $W^{\sigma}$ be the corresponding continuation value. Recall that $W_{k}^{\sigma}$ is the relative gain in continuation payoff for player $k$ evalu ated in $t$ from having $R$ instead of $L$ as $t+1$ status quo. The status quo in $t+1$ affects the outcome in $t+1$ only if players' votes disagree in that period. By stage undomination, this happens when $\theta(t+1) \in D\left(T, V\left(W^{\sigma}\right)\right)$ for some $T \subset \Theta$ (see the explanation in the notation subsection). For such realizations of $\theta(t+1)$, either status quo stays in place, so that the gain in continuation payoff of having status quo $R$ relative to $L$ is $V_{k}\left(W^{\sigma}, \theta(t+1)\right)$. This means that $W^{\sigma}$ is the expectation of $V_{k}\left(W^{\sigma}, \theta(t+1)\right)$ over all disagreement states $\theta(t+1) \in D\left(T, V\left(W^{\sigma}\right)\right)$, condi tional on $\theta(t) \quad \theta$; so from (A3), $W^{\sigma} \quad \Omega^{T}\left(V\left(W^{\sigma}\right)\right)$.

Reciprocally, let $W \in \mathcal{F}^{2}$ be such that $W \quad \Omega^{T}(V(W))$ for some $T \subset \Theta$. Consider the following Markov strategy profile $\sigma$ : for all $\theta \in \Theta$, (i) player $k$ votes for $R$ if $U_{k}(\theta)+\delta W_{k}(\theta)>0$ and for $L$ when $U_{k}(\theta)+\delta W_{k}(\theta)<0$; (ii) if $U_{k}(\theta)+\delta W_{k}(\theta)$ 0 , then player $k$ votes for a different alternative than her opponent if and only if $\theta \in T$. Hence, $\sigma$ prescribes players to vote for different alternatives in state $\theta$ if and only if $\theta \in D(T, V(W))$. By definition, the corresponding continuation value $W^{\sigma}$ is given by the expectation of $V(W, \theta(t+1))$ conditional on $\theta(t)$ over all the states in which players' votes disagree. Therefore, for all $\theta \in \Theta$,

$$
W_{k}^{\sigma}(\theta) \quad \int_{\zeta \in D(T, V(W))}\left[U_{k}(\zeta)+\delta W_{k}^{\sigma}(\zeta)\right] d P_{\theta}(\zeta)
$$

Equation (A4) can be seen as a functional equation in $W^{\sigma}$, and since $W$ $\Omega^{T}(V(W))$, this equation is given by a solution to $W^{\sigma} \quad W$. The right hand side of (A4) is a $\delta$ contraction in $W^{\sigma}$ for the sup norm. Therefore, (A4) has a unique solu tion, which implies that $W^{\sigma} \quad W$. So by construction of $\sigma, \sigma$ prescribes each player $k$ to vote for $R$ when $U_{k}(\theta)+\delta W_{k}^{\sigma}(\theta)>0$ and for $L$ when $U_{k}(\theta)+\delta W_{k}^{\sigma}(\theta)<0$, which means that both players use stage undominated strategies. So $\sigma$ is an equi librium. QED

Lemma 2. For all $T \subseteq \Theta$ and all $f, g \in \mathcal{F}^{2}$ such that $f(\leq, \geq) g(\leq, \geq)\left(0^{\mathcal{F}}, 0^{\mathcal{F}}\right)$, the map $\Omega^{T} \circ V$ is monotonic in the sense that $\left(\Omega^{T} \circ V\right)(f)(\leq, \geq)\left(\Omega^{T} \circ V\right)(g)$.

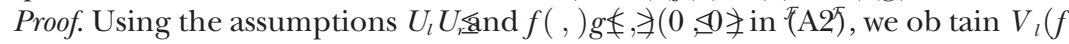
)$\leq V_{l}(g) \leq V_{r}(g) \leq V_{r}(f)$. Using the latter inequalities in (A1), 
we obtain that $D^{\prime \prime}(V(f)) D^{\prime \prime}(V(g)) \varnothing$ and that $D^{\prime}(V(g)) \cup(I(g) \cap T)$ is included in $D(V(f)) \cup(I(f) \cap T)$. From (A3),

$$
\begin{aligned}
\left(\Omega_{r}^{T} \circ V\right)(f, \theta) \quad\left(\Omega_{r}^{T} \circ V\right)(g, \theta) \quad & \int_{D^{\prime}(V(f)) \cup(I(f) \cap T)} V_{r}\left(f_{r}, \zeta\right) d P_{\theta}(\zeta) \\
& \int_{D^{\prime}(V(g)) \cup(I(g) \cap T)} V_{r}\left(g_{r}, \zeta\right) d P_{\theta}(\zeta) .
\end{aligned}
$$

Since the first integral on the right hand side of the above equality integrates larger and positive values over a larger set than the second integral, the left hand side must be positive. An analogous proof shows that $\left(\Omega_{l}^{T} \circ V\right)(f, \theta) \leq\left(\Omega_{l}^{T} \circ V\right)(g, \theta)$. QED

\section{Proofs for Section III.C}

Proof of Proposition 1

Let $W$ be the continuation value of some equilibrium. From lemma $1, W$ $\Omega^{T}(V(W))$ for some $T \subset \Theta$. Using the notations (A2) and (A3), this means that for all $\theta \in \Theta$,

$$
\begin{gathered}
W_{r}(\theta) \quad W_{l}(\theta) \quad \int_{D(T, V(W))}\left[U_{r}(\zeta) \quad U_{l}(\zeta)+\delta\left(W_{r}(\zeta)\right.\right. \\
\left.\left.W_{l}(\zeta)\right)\right] d P_{\theta}(\zeta) .
\end{gathered}
$$

Using the assumption that $U_{r} \quad U_{l} \geq 0$, (A5) implies that for all $\theta \in \Theta, W_{r}(\theta)$ $W_{l}(\theta) \geq 0 .{ }^{22}$ Substituting the last two inequalities in (A2), we obtain $V_{r}\left(W_{r}\right) \geq$ $V_{l}\left(W_{l}\right)$. From (A1), this implies that $D^{\prime \prime}(V(W)) \quad \varnothing$, so $D(T, V(W))$ $D^{\prime}(V(W)) \cup(I(V(W)) \cap T)$. Moreover, from (A1), for all $\zeta \in D^{\prime}(V(W)) \cup$ $(I(V(W)) \cap T), V_{l}(W, \zeta) \leq 0 \leq V_{r}(W, \zeta)$. From (A3), this shows that $\Omega^{T}(V(W))(\leq$, $\geq) 0$, and thus $W(\leq, \geq) 0$. QED

\section{Proof of Proposition 2}

Define the sequence $\left(W^{z}\right)_{z \in \mathbb{N}}$ recursively as follows: for all $\theta \in \Theta, W^{0}(\theta) \quad\left(0^{\mathcal{F}}, 0^{\mathcal{F}}\right)$, and for all $z \geq 0, W^{z+1} \quad\left(\Omega^{\varnothing} V\right)\left(W^{z}\right)$. Below, we show that $\left(W^{z}\right)_{z \in \mathbb{N}}$ is monotonic, is bounded, and thus converges (steps 1 and 2); its limit is the continuation value of a least polarized equilibrium (steps 3 6), and this equilibrium is Pareto best (step 7). Throughout, $W$ is an arbitrary mapping such that $W(\leq, \geq)\left(\Omega^{\varnothing} V\right)(W)$ and $W(\leq, \geq)\left(0^{\mathcal{F}}, 0^{\mathcal{F}}\right) \cdot{ }^{23}$

Step 1: For all $z \in \mathbb{N}, W(\leq, \geq) W^{z+1}(\leq, \geq) W^{z}$.

We prove this by induction on $z$. Since $W^{0} \quad\left(0^{\mathcal{F}}, 0^{\mathcal{F}}\right), V\left(W^{0}\right) \quad U$, and since $U_{l} \leq U_{r}, V_{l}\left(W^{0}\right) \leq V_{r}\left(W^{0}\right)$. Using the latter inequality in (A1), we obtain

${ }^{22}$ To see this, let $w \stackrel{\circ}{=} \inf _{\theta \in \Theta}\left(W_{r}(\theta)-W_{l}(\theta)\right)$ and let $\left(\theta_{n}\right)_{n \in \mathbb{N}}$ be a sequence of states such that $W_{r}\left(\theta_{n}\right)-W_{l}\left(\theta_{n}\right) \rightarrow w$. Then from (A5), for all $n \in \mathbb{N}, W_{r}\left(\theta_{n}\right)-W_{l}\left(\theta_{n}\right) \geq \delta w P_{\theta_{n}}(D(T, V(W)))$. Since $0 \leq P_{\theta_{n}} \leq 1$, there exists a subsequence along which $\left(P_{\theta_{n}}(D(T, V(W)))\right)_{n \in \mathbb{N}}$ converges to some $p \in[0,1]$. Taking the limit along this subsequence, we obtain $w \geq \delta w p$, so $w \geq 0$, as needed.

${ }^{23}$ Such a mapping exists, e.g., the greatest element of $\mathcal{F}^{2}$ in the order $(\leq, \geq)$ : for all $\theta \in \Theta$, $\bar{W}(\theta) \stackrel{\bullet}{=}(-m /(1-\delta), m /(1-\delta))$. The reason why we allow $\bar{W}$ to be any such mapping will be clear in the proofs of propositions 3 and 7 . 
$D^{\prime \prime}\left(V\left(W^{0}\right)\right) \quad \varnothing$, and thus $D\left(\varnothing, V\left(W^{0}\right)\right) \quad D^{\prime}\left(V\left(W^{0}\right)\right), \quad$ so for all $\theta \in$ $D\left(\varnothing, V\left(W^{0}\right)\right), V_{l}\left(W^{0}, \theta\right) \leq 0 \leq V_{r}\left(W^{0}, \theta\right)$. Using the latter inequality in (A3), we obtain $\left(\Omega^{\varnothing} \circ V\right)\left(W^{0}\right)(\leq, \geq)\left(0^{\mathcal{F}}, 0^{\mathcal{F}}\right)$ or, equivalently, $W^{1}(\leq, \geq) W^{0}$. Moreover, since $W(\leq, \geq)\left(0^{\mathcal{F}}, 0^{\mathcal{F}}\right) \quad W^{0}$, lemma 2 implies that $\left(\Omega^{\varnothing} V\right)(W)(\leq, \geq)\left(\Omega^{\varnothing} V\right)\left(W^{0}\right)$, which, together with the assumption that $W(\leq, \geq)\left(\Omega^{\varnothing} V\right)(W)$, implies that $W(\leq, \geq) W^{1}$. This completes the proof of step 1 for $z \quad 0$. Suppose now that $W(\leq, \geq) W^{z}(\leq, \geq) \cdots$ $(\leq, \geq) W^{0}$ for some $z \geq 0$. Then lemma 2 implies that $\left(\Omega^{\varnothing} V\right)(W)(\leq, \geq) W^{z+1}$ $(\leq, \geq) W^{z}$, which, together with $W(\leq, \geq)\left(\Omega^{\varnothing} \circ V\right)(W)$, completes the induction ar gument.

Step 2: The sequence $\left(W^{z}\right)_{z \in \mathbb{N}}$ converges pointwise to some $W^{\infty}$ such that $W(\leq, \geq)$ $W^{\infty}$.

From step 1, for all $\theta \in \Theta$, the sequence $\left(W_{k}^{z}\right)(\theta)$ is bounded and increasing (decreasing) in $z$ for $k \quad r\left(\begin{array}{ll}k & l\end{array}\right)$. As such, it converges to some $W_{k}^{\infty}(\theta)$. The in equality $W(\leq, \geq) W^{\infty}$ follows directly from step 1 .

Step 3: If $1_{S}$ denotes the indicator function of a set $S \subseteq \Theta$, then $1_{D\left(\varnothing, V\left(W^{*}\right)\right)}$ is in creasing in $z$ and converges pointwise to $1_{D\left(\varnothing, V\left(W^{*}\right)\right)}$.

Since $W^{z}(\leq, \geq) W^{0}$, the same reasoning as in step 1 shows that for all $z \in \mathbb{N} \cup\{\infty\}$, $D\left(\varnothing, V\left(W^{z}\right)\right) \quad D^{\prime}\left(V\left(W^{z}\right)\right)$, so $1_{D\left(\varnothing, V\left(W^{z}\right)\right)} \quad 1_{D^{\prime}\left(V\left(W^{*}\right)\right)}$. From (A2) and step $1, V\left(W^{z}\right)$ is increasing in $z$ for the order $(\leq, \geq)$, so from (A1), $1_{D^{\prime}\left(V\left(W^{*}\right)\right)}$ is increasing. Now let $\theta$ be such that $1_{D^{\prime}\left(V\left(W^{\circ}\right)\right)}(\theta) \quad 1$. Then $V_{l}\left(W^{\infty}\right)(\theta)<0<V_{r}\left(W^{\infty}\right)$.

Since $V$ is continuous in $W$, for $z$ sufficiently large, $V_{l}\left(W^{z}\right)(\theta)<0<V_{r}\left(W^{z}\right)(\theta)$, so $\lim _{z \rightarrow \infty} 1_{D^{\prime}\left(V\left(W^{*}\right)\right)}(\theta) \quad 1$. Reciprocally, suppose $\lim _{z \rightarrow \infty} 1_{D^{\prime}\left(V\left(W^{*}\right)\right)}(\theta) \quad 1$. Then for some $z, V_{l}\left(W^{z}\right)(\theta)<0<V_{r}\left(W^{z}\right)(\theta)$, and since $V\left(W^{z}\right)$ is increasing in $z$ for the or $\operatorname{der}(\leq, \geq), V_{l}\left(W^{\infty}\right)(\theta)<0<V_{r}\left(W^{\infty}\right)$, so $1_{D^{\prime}\left(V\left(W^{\infty}\right)\right)}(\theta) \quad 1$.

Step 4: $W^{\infty}$ is a fixed point of $\Omega^{\varnothing} \vee V$.

For all $z \in \mathbb{N}, k \in\{l, r\}$, and $\theta \in \Theta$,

$$
\begin{aligned}
W_{k}^{z+1}(\theta) & \left(\Omega^{\varnothing} V\right)_{k}\left(W^{z}\right)(\theta) \\
& \int_{\zeta \in \Theta} 1_{D\left(\varnothing, V\left(W^{z}\right)\right.}(\zeta) \times\left[U_{k}(\theta)+W_{k}^{z}(\zeta)\right] d P_{\theta}(\zeta) .
\end{aligned}
$$

From step 2, the first term of the above equation tends to $W^{\infty}(\theta)$ as $z \rightarrow \infty$. Using steps 2 and 3 together with Lebesgue's monotone convergence theorem, the last term tends to $\left(\Omega^{\varnothing} \circ\right)\left(W^{\infty}\right)(\theta)$.

Step 5: For any equilibrium $\sigma, W^{\sigma}(\leq, \geq) W^{\infty}$.

From lemma 1, there exists $T \subseteq \Theta$ such that $W^{\sigma} \quad\left(\Omega^{T} \circ V\right)\left(W^{\sigma}\right)$ and players' votes disagree in $\sigma$ when $\theta \in D\left(T, V\left(W^{\sigma}\right)\right)$. As shown in the proof of proposition 1, in all disagreement states $\theta \in D\left(T, V\left(W^{\sigma}\right)\right), V_{l}\left(W^{\sigma}, \theta\right) \leq 0 \leq V_{r}\left(W^{\sigma}, \theta\right)$. Substi tuting this inequality and $D\left(\varnothing, V\left(W^{\sigma}\right)\right) \subseteq D\left(T, V\left(W^{\sigma}\right)\right)$ into (A3), we obtain $\left(\Omega^{T} \circ V\right)\left(W^{\sigma}\right)(\leq, \geq)\left(\Omega^{\varnothing} V\right)\left(W^{\sigma}\right)$, and thus $W^{\sigma}(\leq, \geq)\left(\Omega^{\varnothing} \vee V\right)\left(W^{\sigma}\right)$. Hence, $W^{\sigma}$ satis fies the condition for being $W$. From step $2, W^{\sigma}(\leq, \geq) W^{\infty}$.

Step 6: $W^{\infty}$ is the continuation value of a least polarized equilibrium $\sigma^{\infty}$ in which players' votes disagree in a smaller set of states (in the inclusion sense) than in any other equilibrium.

Step 4 and lemma 1 imply that $W^{\infty}$ is the continuation value of some equilib rium $\sigma^{\infty}$, and from step $5, \sigma^{\infty}$ is less polarized than any other equilibrium $\sigma$. Let $\theta \in \Theta$ be a state in which players' votes disagree in $\sigma^{\infty}$. As established in the proof of proposition $1, V_{l}\left(W^{\infty}, \theta\right) \leq 0 \leq V_{r}\left(W^{\infty}, \theta\right)$, and from lemma $1, \theta \in D\left(\varnothing, V\left(W^{\infty}\right)\right)$, so necessarily, $V_{l}\left(W^{\infty}, \theta\right)<0<V_{r}\left(W^{\infty}, \theta\right)$. Since $W^{\sigma}(\leq, \geq) W^{\infty}$, this implies that 
$V_{l}\left(W^{\sigma}, \theta\right)<0<V_{r}\left(W^{\sigma}, \theta\right)$, and stage undomination implies that players' votes al so disagree in $\sigma$ in state $\theta$.

Step 7: $\sigma^{\infty}$ is Pareto better than any other equilibrium $\sigma$.

Suppose that both players deviate from $\sigma^{\infty}$ to the behavior prescribed by $\sigma$ only in $t \quad 0$. From step 6 , players disagree in more states under $\sigma$ than under $\sigma^{\infty}$. Therefore, the deviation from $\sigma^{\infty}$ to $\sigma$ in $t \quad 0$ can change the outcome at $t \quad 0$ only if under $\sigma^{\infty}$ players agree to change the status quo while under $\sigma$ the status quo remains. Suppose without loss of generality that $q(0) \quad R$, and hence $L$ is implemented under $\sigma^{\infty}$ while $R$ stays in place under $\sigma$. Since players play their equilibrium strategy $\sigma^{\infty}$ in the subgame starting in $t \quad 1$, the net effect of this deviation for player $k$ is $U_{k}(\theta(0))+\delta W_{k}^{\infty}(\theta(0))$. Since both players vote for $L$ un $\operatorname{der} \sigma^{\infty}, U_{k}(\theta(0))+\delta W_{k}^{\infty}(\theta(0))$ must be nonpositive for both $k \in\{l, r\}$, which implies that the deviation weakly decreases the payoff of both players. Consider now the strategy profile in which players play $\sigma$ in $t \quad 0$ and $\sigma^{\infty}$ afterward, and let players deviate from that profile by playing according to $\sigma$ also in $t$. The same reason ing as above shows that this deviation further decreases the payoffs of both players irrespective of the status quo distribution in $t \quad 1$. By induction on the number of periods in which players deviate from $\sigma^{\infty}$ to $\sigma$, step 7 follows.

Step 8: A most polarized equilibrium exists and is Pareto worst.

The proof follows the same logic as steps 17 with the sequence $\left(W^{z}\right)_{z \in \mathbb{N}}$ de fined recursively as follows: $W^{0}$ is the greatest element of $\mathcal{F}$ (i.e., $W(\theta)$ $\left.\left(\begin{array}{ll}m /(1 & \delta\end{array}\right), m /\left(\begin{array}{ll}1 & \delta\end{array}\right)\right)$ for all $\left.\theta \in \Theta\right)$, and for all $z \geq 0, W^{z+1} \quad\left(\Omega^{\Theta} \circ V\right)\left(W^{z}\right)$. We omit the details for brevity. QED

\section{Proofs for Section III.D}

Proof of Proposition 3

Let $V$ and $V^{\prime}$ refer to the maps defined in (A2) and let $\sigma$ and $\sigma^{\prime}$ be least polarized equilibria corresponding to $(U, \delta)$ and to $\left(U^{\prime}, \delta^{\prime}\right)$, respectively, as constructed in the proof of proposition 2. As shown in step 2 of that proof, $W(\leq, \geq) W^{\sigma}$ for any $W$ such that (i) $W(\leq, \geq)\left(0^{\mathcal{F}}, 0^{\mathcal{F}}\right)$ and (ii) $W(\leq, \geq)\left(\Omega^{\varnothing} V\right)(W)$. Hence, to prove proposition 3, it suffices to show that parts i and ii are satisfied for $W \quad W^{\sigma^{\prime}}$. In equality i follows from proposition 1 . To prove ii, note that as shown in the proof of proposition 2, $W^{\sigma^{\prime}} \quad\left(\Omega^{\varnothing} \circ V^{\prime}\right)\left(W^{\sigma^{\prime}}\right)$. Moreover, from (A2),

$$
V^{\prime}\left(W^{\sigma^{\prime}}\right) \quad V\left(\frac{\delta^{\prime}}{\delta} W^{\sigma^{\prime}}+\frac{1}{\delta}\left(\begin{array}{ll}
U^{\prime} & U
\end{array}\right)\right),
$$

and since $\delta^{\prime}>\delta$ and $U^{\prime}(\leq, \geq) U$,

$$
\left[\frac{\delta^{\prime}}{\delta} W^{\sigma^{\prime}}+\frac{1}{\delta}\left(U^{\prime} \quad U\right)\right](\leq, \geq) W^{\sigma^{\prime}}(\leq, \geq)\left(0^{\mathcal{F}}, 0^{\mathcal{F}}\right) .
$$

Together with lemma 2, this implies that

$$
\begin{array}{ll}
W^{\sigma^{\prime}} \quad\left(\Omega^{\varnothing} \circ V^{\prime}\right)\left(W^{\sigma^{\prime}}\right) \\
\\
\left(\Omega^{\varnothing} V\right)\left[\frac{\delta^{\prime}}{\delta} W^{\sigma^{\prime}}+\frac{1}{\delta}\left(U^{\prime} \quad U\right)\right](\leq, \geq)\left(\Omega^{\varnothing} V\right)\left(W^{\sigma^{\prime}}\right) .
\end{array}
$$


The proof for the most polarized equilibrium follows the same steps and is omit ted for brevity. QED

\section{Proof of Proposition 4}

Suppose that $\sigma_{l}^{g}$ is not a best response for player $l$ to $\sigma_{r}^{g}$. From the one step deviation principle, this is true if and only if for some $\theta \in \Theta$, conditional on $\theta(0) \quad \theta$, player $l$ is better off voting for $R$ than for $L$ at $t \quad 0$, given that she ex pects $\sigma^{g}$ to be played in all future periods. By definition of $\sigma^{g}$, this is equivalent to saying that for $\theta(0) \quad \theta$, player $l$ is better off with alternative $R$ in all periods than with alternative $L$ in all periods. Hence, $\sigma_{l}^{g}$ is not a best response for player $l$ to $\sigma_{r}^{g}$ if and only if condition (3) is violated for player $l$. A symmetric argument for player $r$ completes the proof. QED

\section{Proof of Corollary 2}

Since $U_{k}(\theta)$ is bounded by $m$ on $\Theta$, for all $T>0$,

$$
\begin{aligned}
& E_{\theta(0)}\left[\sum_{t=0}^{\infty} \delta^{t} U_{l}(\theta(t))\right] \quad E_{\theta(0)}\left[\sum_{t=0}^{T} \delta^{t}\left(U_{l}(\theta(t)) \quad E^{*}\left[U_{l}(\tilde{\theta})\right]\right)\right. \\
& \left.+\sum_{t=T}^{\infty} \delta^{t}\left(U_{l}(\theta(t)) \quad E\left[U_{l}(\tilde{\theta})\right]\right)\right]+\frac{E\left[U_{l}(\tilde{\theta})\right]}{1 \quad \delta} \\
& \leq \frac{2 m\left(1 \quad \delta^{T}\right)}{1 \delta}+\sum_{t=T}^{\infty} \delta^{t}\left|E_{\theta(0)}\left[U_{l}(\theta(t))\right] \quad E\left[U_{l}(\tilde{\theta})\right]\right|+\frac{E\left[U_{l}(\tilde{\theta})\right]}{1 \delta} .
\end{aligned}
$$

Since $E\left[U_{l}(\tilde{\theta})\right] \neq 0$, by definition of uniform ergodicity, one can choose $T$ large enough such that for all $t \geq T$ and all $\theta \in \Theta,\left|E_{\theta(0)}{ }_{\theta}\left[U_{l}(\theta(t))\right] \quad E\left[U_{l}(\tilde{\theta})\right]\right|<$ $\left|E\left[U_{l}(\tilde{\theta})\right]\right| / 2$. Moreover, there exists $\delta_{l}<1$ such that for all $\delta \in\left[\delta_{l}, 1\right), 2 m\left(1 \quad \delta^{T}\right)<$ $\left|E\left[U_{l}(\tilde{\theta})\right]\right| / 2$. Substituting these bounds in the above inequality, we obtain that for all $\delta \geq \delta_{l}$ and all $\theta \in \Theta$,

$$
\begin{aligned}
E_{\theta(0) \theta}\left[\sum_{t=0}^{\infty} \delta^{t} U_{l}(\theta(t))\right]< & \frac{\left|E\left[U_{l}(\tilde{\theta})\right]\right|}{2(1 \quad \delta)}+\left(\sum_{t=T}^{\infty} \delta^{t}\right) \frac{\left|E\left[U_{l}(\tilde{\theta})\right]\right|}{2} \\
& +\frac{E\left[U_{l}(\tilde{\theta})\right]}{1 \quad \delta}<0 .
\end{aligned}
$$

A symmetric argument holds for the rightist player for some threshold $\delta_{r}<1$. Setting $\delta \quad \max \left\{\delta_{l}, \delta_{r}\right\}$ completes the proof. QED

\section{B. Proofs for the NAlternative Model}

For the sake of brevity, we outline here only the main steps of the arguments. The omitted steps can be found in the online appendix. 
Notation. The set of Markov states of the game $\Gamma_{c}^{\mathrm{en}}$, denoted by $S$, comprises three kinds of states: direction states, in which the proposer must choose a direc tion; voting states, in which players vote simultaneously; and payoff states, in which players have no actions to choose, the current agreement is implemented, and players receive the corresponding payoff. A typical direction state and payoff state of $\Gamma_{c}^{\mathrm{en}}$ are denoted by $\langle\theta, x\rangle$ and $\langle\langle\theta, x\rangle\rangle$, respectively, where $\theta \in \Theta$ is the period specific state of nature and $x \in X$ is the current agreement or the final agreement, respectively. A typical voting state is denoted by $\left\langle\theta, x, x^{\prime}\right\rangle$, where $\theta$ and $x$ have the same interpretation as in $\langle\theta, x\rangle$, and $x^{\prime}$ is the proposal under con sideration. For any Markov strategy profile $\sigma$ and any state $s \in S, V_{k}^{\sigma}(s)$ denotes the expected continuation payoff of player $k$ from state $s$ given continuation play $\sigma$, and $\phi^{\sigma}(s)$ denotes the (possibly stochastic) outcome in a given period, starting from state $s$ and given continuation play $\sigma$.

The following lemma formalizes the intuition laid out in Section IV.A that the endogenous status quo has a polarizing effect whenever the bargaining proce dure is such that in each period the outcome is monotonic in the status quo.

Lemma 3. Let $\sigma$ be an equilibrium of $\Gamma_{c}^{\text {en }}$. If for all $\theta \in \Theta$ and $P_{\theta}$ almost all $\zeta \in$ $\Theta$, the distribution of $\left(\phi^{\sigma}(\langle\zeta, q\rangle)\right)_{q \in X}$ is monotonic in $q$ in the first order stochastic dominance sense, then $\sigma$ is polarized. ${ }^{24}$

Proof. Since the distribution of the outcome is almost always monotonic in the status quo and since the outcome in a given period becomes the status quo in the next period, a straightforward induction shows that for all $\theta \in \Theta$, conditional on $\theta(t) \quad \theta$, for all $t^{\prime} \geq t+1$ and almost all realizations of $\theta\left(t^{\prime}\right)$, the distribution of the outcome in $t^{\prime}$ is monotonic in the status quo in period $t+1$. Since $U_{r}$ is more rightist than $U_{l}$, this implies that for all $\theta \in \Theta$ and all $x_{n}, x_{n+1} \in X, W_{r}^{\sigma}\left(\theta, x_{n+1}\right)$ $W_{r}^{\sigma}\left(\theta, x_{n}\right) \geq W_{l}^{\sigma}\left(\theta, x_{n+1}\right) \quad W_{l}^{\sigma}\left(\theta, x_{n}\right)$. Since for any direction state $\langle\zeta, x\rangle, V^{\sigma}(\langle\zeta$, $x\rangle) \quad E_{\phi^{\sigma}}\left[U\left(\phi^{\sigma}(\langle\zeta, q\rangle)\right)+\delta W^{\sigma}\left(\phi^{\sigma}(\langle\zeta, q\rangle)\right)\right]$, the previous inequality implies that for all $\zeta \in \Theta$ such that $\left(\phi^{\sigma}(\langle\zeta, q\rangle)\right)_{q \in X}$ is monotonic in $q$,

$$
V_{r}^{\sigma}\left(\left\langle\zeta, x_{n+1}\right\rangle\right) \quad V_{r}^{\sigma}\left(\left\langle\zeta, x_{n}\right\rangle\right) \geq V_{l}^{\sigma}\left(\left\langle\zeta, x_{n+1}\right\rangle\right) \quad V_{l}^{\sigma}\left(\left\langle\zeta, x_{n}\right\rangle\right) .
$$

It is easy to show (online appendix claim 1) that if $\sigma$ is an equilibrium, $V_{k}^{\sigma}(\langle\zeta$, $\left.\left.x_{n+1}\right\rangle\right) \quad V_{k}^{\sigma}\left(\left\langle\zeta, x_{n}\right\rangle\right)$ cannot have the same strict sign for both players. Together with the above inequality, this implies that $V_{k}^{\sigma}\left(\left\langle\zeta, x_{n+1}\right\rangle\right) \quad V_{k}^{\sigma}\left(\left\langle\zeta, x_{n}\right\rangle\right)$ is weakly pos itive (negative) for $k \quad r(k \quad l)$. Since $W^{\sigma}(\theta, q) \quad \int_{\zeta \in \Theta} V_{k}^{\sigma}(\langle\zeta, q\rangle) d P_{\theta}(\zeta)$, this implies that $W_{k}^{\sigma}(\theta, q)$ is weakly increasing (decreasing) in $q$ for $k \quad r\left(\begin{array}{ll}k & l\end{array}\right)$, as needed. QED

Proof of Proposition 5

For all Markov strategy profiles $\sigma$ of $\Gamma_{c}^{\mathrm{en}}, k \in\{l, r\}$, and $\theta \in \Theta$, let $\sigma_{k}(\theta)$ and $\sigma_{k}(\theta)$ denote the restriction of $\sigma_{k}$ to the Markov states in which the state of

${ }^{24}$ That is, for all $q^{\prime}>q$ and all $x \in X$, the probability that the outcome is to the right of $x$ is weakly greater under $\phi^{\sigma}\left(\left\langle\zeta, q^{\prime}\right\rangle\right)$ than under $\phi^{\sigma}(\langle\zeta, q\rangle)$. 
nature is $\theta$ and to those in which the state of nature is different from $\theta$, respec tively. Let $k^{\prime}$ denote the opponent of $k$. For a given $\sigma_{k}(\theta)$ and $\sigma_{k^{\prime}}$, we say that $\sigma_{k}^{*}(\theta)$ is a stage undominated best response to $\sigma_{k}(\theta)$ and $\sigma_{k^{\prime}}$ if $\sigma_{k}^{*}(\theta)$ prescribes stage undominated actions in all Markov states with state of nature $\theta$ given con tinuation play $\left(\sigma_{k}^{*}(\theta), \sigma_{k}(\theta), \sigma_{k^{\prime}}\right)$ in $\Gamma_{c}^{\text {en }}$. Let $\Sigma_{k}^{\sigma}(\theta)$ denote the set of such stage undominated best responses. Note that $\Sigma_{k}^{\sigma}(\theta)$ depends on $\sigma$ only through $\sigma_{k}(\theta)$ and $\sigma_{k^{\prime}}$. If we denote $\Sigma^{\sigma} \stackrel{\ominus}{=}\left(\Sigma_{l}^{\sigma}(\theta), \Sigma_{r}^{\sigma}(\theta)\right)_{\theta \in \Theta}$, then by construction of $\Sigma^{\sigma}$, a strategy profile $\sigma$ is an equilibrium of $\Gamma_{c}^{\mathrm{en}}$ if and only if $\sigma \in \Sigma^{\sigma}$.

If $k$ is the proposer when the state of nature is $\theta$, then $\sigma_{k}^{*}(\theta) \in \Sigma_{k}^{\sigma}(\theta)$ is called monotonic if $\left(\phi^{\sigma_{k}^{*}(\theta), \sigma_{k}(\theta)}(\langle\theta, q\rangle)\right)_{q \in X}$ is monotonic in $q$ in the sense of lemma 3. Let $\hat{\Sigma}_{k}^{\sigma}(\theta) \subseteq \Sigma_{k}^{\sigma}(\theta)$ denote the set of monotonic, stage undominated best responses. If $k$ is not the proposer in state $\theta$, we simply set $\hat{\Sigma}_{k}^{\sigma}(\theta) \doteq \Sigma_{k}^{\sigma}(\theta)$. From lemma 3 , to prove the existence of a polarized equilibrium, it suffices to show that the correspon dence $\sigma \rightarrow \hat{\Sigma}^{\sigma}$ has a fixed point. However, one cannot resort to the usual fixed point theorems based on continuity and convex valuedness, because $\hat{\Sigma}^{\sigma}$ is typically not convex. To circumvent this problem, we use the following theorem due to Eilenberg and Montgomery (1946, theorem 1)..$^{25}$

Theorem 1. If $C$ is a compact and convex subset of a Euclidean space and $F$ : $C \rightarrow 2^{C}$ is an upper hemicontinuous, contractible valued correspondence, then $F$ has a fixed point.

In our case, $C$ is the set of Markov strategy profiles of $\Gamma_{c}^{\mathrm{en}}$. Since $\Theta$ is finite, $C$ satisfies the conditions of the theorem. Thus, to complete the proof, it suffices to show that $\sigma \rightarrow \hat{\Sigma}^{\sigma}$ is upper hemicontinuous and contractible valued. The former property follows from standard continuity arguments.

We now sketch the argument for the contractibility of $\hat{\Sigma}^{\sigma}$; the detailed proof can be found in claim 2 in the online appendix. By the standard argument, for all $\sigma, \theta \in \Theta$, and $k \in\{l, r\}$, there exists $\sigma_{k}^{*}(\theta) \in \Sigma_{k}^{\sigma}(\theta)$. Moreover, $\sigma_{k}^{\prime}(\theta) \in \Sigma_{k}^{\sigma}(\theta)$ if and only if it differs from $\sigma_{k}^{*}(\theta)$ at most in Markov states in which player $k$ is indifferent between the two possible continuation states, given continuation play $\left(\sigma_{k}^{*}(\theta)\right.$, $\left.\sigma_{k}(\theta), \sigma_{k^{\prime}}\right)$. Hence, one can find $\sigma_{k}^{*}(\theta) \in \Sigma_{k}^{\sigma}(\theta)$ such that in any voting state and di rection state in which $k$ is indifferent between the continuation states, $\sigma_{k}^{*}(\theta)$ pre scribes $k$ to play no and left, respectively. We prove that $\sigma_{k}^{*}(\theta)$ is monotonic, and thus $\sigma_{k}^{*}(\theta) \in \hat{\Sigma}^{\sigma}(\theta)$.

By definition, $\hat{\Sigma}_{k}^{\sigma}(\theta)$ is contractible if there exists $a>0, \sigma_{k}(\theta) \in \hat{\Sigma}_{k}^{\sigma}(\theta)$, and a con tinuous function $f:[0, a] \times \hat{\Sigma}_{k}^{\sigma}(\theta) \rightarrow \hat{\Sigma}_{k}^{\sigma}(\theta)$ such that for all $\sigma_{k}^{\prime}(\theta) \in \hat{\Sigma}^{\sigma}(\theta), f\left(0, \sigma_{k}^{\prime}(\theta)\right)$ $\sigma_{k}^{\prime}(\theta)$ and $f\left(a, \sigma_{k}^{\prime}(\theta)\right) \quad \sigma_{k}(\theta)$. We use $a \quad 2, \sigma_{k}(\theta) \quad \sigma_{k}^{*}(\theta)$, and the following func tion $f$ : for any $\sigma_{k}^{\prime}(\theta) \in \hat{\Sigma}^{\sigma}$, as $a$ increases from zero to one, the probability with which $f\left(a, \sigma_{k}^{\prime}(\theta)\right)$ prescribes $k$ to play no when indifferent increases continuously to one, and as $a$ increases from one to two, the probability with which $f\left(a, \sigma_{k}^{\prime}(\theta)\right)$ prescribes $k$ to play left when indifferent increases continuously to one. We show

${ }_{25}$ This result is actually a corollary from Eilenberg and Montgomery (1946, theorem 1); see Reny $(2011,503)$. In Reny's paper, $C$ is an absolute retract, but any convex subset of a Euclidean space is an absolute retract, and $F$ is also required to be nonempty valued; but this is implied by $F$ being contractible valued. 
that $f$ transforms any $\sigma_{k}^{\prime}(\theta)$ into $\sigma_{k}^{*}(\theta)$ while preserving the best response and mono tonicity properties of $\sigma_{k}^{\prime}(\theta)$. QED

\section{Proof of Proposition 6}

Let $\sigma$ be an equilibrium of $\Gamma_{c}^{\text {en }}$. Assumption 1(ii) implies that $W^{\sigma}(\theta, q)$ depends on $\theta$ only via $\theta_{1}$. Since $V(\langle\langle\zeta, x\rangle\rangle) \quad U(\zeta, x)+\delta W^{\sigma}\left(\zeta_{1}, q\right)$, assumption 1 (i) implies that for all $\theta \in \Theta$ and $P_{\theta}$ almost all $\zeta$, the preferences induced by $(V(\langle\langle\zeta, x\rangle\rangle))_{x \in X}$ are strict, so the outcome $\phi^{\sigma}(\langle\zeta, q\rangle)$ is deterministic and monotonic in $q$. Deter minism follows from a simple backward induction starting from the states $\langle\zeta$, $\left.x_{2}, x_{1}\right\rangle$ and $\left\langle\zeta, x_{N}{ }_{1}, x_{N}\right\rangle$. To show monotonicity, suppose by contradiction that for some $x_{n}, x_{n+1} \in X, \phi^{\sigma}\left(\left\langle\zeta, x_{n}\right\rangle\right)>\phi^{\sigma}\left(\left\langle\zeta, x_{n+1}\right\rangle\right)$. This implies that $\phi^{\sigma}\left(\left\langle\zeta, x_{n}\right\rangle\right)>x_{n}$ or $\phi^{\sigma}\left(\left\langle\zeta, x_{n+1}\right\rangle\right)<x_{n+1}$. Suppose the former; the proof in the latter case is identical. Then the proposer can obtain $\phi^{\sigma}\left(\left\langle\zeta, x_{n}\right\rangle\right)$ by playing right in $\left\langle\zeta, x_{n+1}\right\rangle$. Since she has strict preferences and $\sigma$ is an equilibrium, this implies that

$$
V_{p r}^{\sigma}\left(\left\langle\left\langle\zeta, \phi^{\sigma}\left(\left\langle\zeta, x_{n+1}\right\rangle\right)\right\rangle\right\rangle\right)>V_{p r}^{\sigma}\left(\left\langle\left\langle\zeta, \phi^{\sigma}\left(\left\langle\zeta, x_{n}\right\rangle\right)\right\rangle\right\rangle\right) .
$$

But she can also obtain the outcome $\phi^{\sigma}\left(\left\langle\zeta, x_{n+1}\right\rangle\right)$ from state $\left\langle\zeta, x_{n}\right\rangle$ by voting no when the path of play reaches $\left\langle\zeta, x_{n+1}, x_{n+2}\right\rangle$ (if $\phi^{\sigma}\left(\left\langle\zeta, x_{n+1}\right\rangle\right.$ ) $x_{n+1}$ ) or by play ing left in $\left\langle\zeta, x_{n}\right\rangle$ and $\sigma$ thereafter (if $\phi^{\sigma}\left(\left\langle\zeta, x_{n+1}\right\rangle\right)<x_{n+1}$ ), a profitable deviation. Lemma 3 implies then that $\sigma$ is polarized. The argument for existence when $\Theta_{1}$ is finite is standard and hence is relegated to the online appendix (see the proof of equilibrium existence for proposition 6). QED

\section{Sketch of the Proof of Proposition 7}

For all $b \in \mathbb{R}^{2}$, let $U^{b}$ denote the profile of quadratic current preferences with biases $b$, and for all finite subsets $X$ of $\mathbb{R}$, let $\Gamma_{c}^{\text {ex }}\left(X, U^{b}\right)$ and $\Gamma_{c}^{\text {en }}\left(X, U^{b}\right)$ denote the game with an exogenous and endogenous status quo, respectively, in which players' cur rent preferences are $U^{b}$ and the set of alternatives is $X$.

For any pair of alternatives $x, y \in \mathbb{R}$, the game $\Gamma_{c}^{\mathrm{en}}\left(\{x, y\}, U^{b}\right)$ is strategically equiv alent to the two alternative game analyzed in Section III.C. Hence, using argu ments similar to those in the proof of proposition 2, one can show that in the least polarizing equilibrium of the game $\Gamma_{c}^{\mathrm{en}}\left(\{x, y\}, U^{b}\right)$, players' intertemporal prefer ences over $\{x, y\}$ coincide ordinally with $U^{b+p^{*}}$ with $p_{l}^{*}<0<p_{r}^{*}$. Moreover, the ran dom walk assumption implies that $p^{*}$ does not depend on $\{x, y\}$ (claim 3 in the online appendix).

Let $\sigma^{\text {ex }}\left(X, U^{b+p^{n}}\right)$ denote the equilibrium of $\Gamma_{c}^{\text {ex }}\left(X, U^{b+p^{n}}\right)$, as described in Sec tion IV.B. One can show (step 2 in the proof of proposition 7 in the online ap pendix) that when $\sigma^{\text {ex }}\left(X, U^{b+p^{n}}\right)$ is played in $\Gamma_{c}^{\mathrm{en}}\left(X, U^{b}\right)$, players' intertemporal preferences over any pair of consecutive alternatives $\{x, y\} \in X$ coincide with their intertemporal preferences in the least polarized equilibrium of $\Gamma_{c}^{\mathrm{en}}\left(\{y, z\}, U^{b}\right)$. Therefore, the intertemporal preferences induced by $\sigma^{\mathrm{ex}}\left(X, U^{b+p^{n}}\right)$ in $\Gamma_{c}^{\mathrm{en}}\left(X, U^{b}\right)$ over consecutive alternatives coincide ordinally with $U^{b+p^{p}}$. The single peakedness of $U^{b+p^{n}}$ implies then that they coincide over the entire $X$. One can then conclude (step 1 in the proof of proposition 7 in the online appendix) that since $\sigma^{\text {ex }}(X$, 
$\left.U^{b+p^{r}}\right)$ is an equilibrium of $\Gamma_{c}^{\mathrm{ex}}\left(X, U^{b+p^{r}}\right)$, this implies that $\sigma^{\mathrm{ex}}\left(X, U^{b+p^{r}}\right)$ is also an equilibrium of $\Gamma_{c}^{\mathrm{en}}\left(X, U^{b}\right)$. QED

\section{Proof for Section IV.C: Sketch of the Proof of Proposition 8}

The intuition for this proof is simple: if in equilibrium $q$ is replaced by $x$ at some bargaining time $t$, then player $k$ can veto this change and stay at $q$ until $x$ replaces $q$ again. If preferences evolve smoothly and players bargain frequently, $x$ will indeed replace $q$ a small instant later with large probability. If $q$ gives player $k$ a higher cur rent payoff than $x$ in a neighborhood of $\theta$, this deviation is profitable.

More formally, suppose by contradiction that, in equilibrium, status quo $q$ is re placed by $x$ in some state $\theta$ such that $u_{k}(\theta, x)>u_{k}(\theta, q)$. By continuity of $u, u_{k}(\zeta, q)>$ $u_{k}(\zeta, x)+v$ for some $v>0$ for all $\zeta \in B^{\prime}$, where $B^{\prime}$ is a neighborhood $\theta$ included in the neighborhood $B$ of the statement of the proposition. Consider the following deviation in some period with $(\theta, q)$ : player $k$ vetoes the change from $q$ to $x$ in that period, and in all consecutive periods she vetoes any move from $q$ until the equi librium strategies prescribe players to replace $q$ by $x$. If, under this deviation, $x$ is implemented before the state exits $B^{\prime}$, then this deviation gives a flow payoff gain of at least $v$ to $k$ before $x$ is implemented and leads to the same path of play after that. Using assumption 2 and the assumption on the behavior of the equilibrium sequence $\left(\sigma^{s}\right)_{s \in \mathbb{N}}$ in $B$, we show in the online appendix that as $\Delta \rightarrow 0$, the probability that the state exits $B^{\prime}$ before $x$ is implemented becomes negligible relative to the payoff gain of the deviation in the other case. QED

\section{References}

Acemoglu, Daron, Georgy Egorov, and Konstantin Sonin. 2008. "Coalition For mation in Non democracies." Rev Econ Studies 75 (4): 9871009.

. 2012. "Dynamics and Stability of Constitutions, Coalitions, and Clubs." A.E.R. 102 (4): 144676.

. 2015. "Political Economy in a Changing World." LPE. 123 (5): 1038 86.

Acemoglu, Daron, and James Robinson. 2001. "A Theory of Political Transi tions.” A.E.R. 91:938 63.

Alesina, Alberto, and Allan Drazen. 1991. "Why Are Stabilizations Delayed?" A.E.R. 81 (5): 117088.

Alesina, Alberto, and Guido Tabellini. 1990. "A Positive Theory of Fiscal Deficits and Government Debt.” Rev Econ Studies 57:403 14.

Anesi, Vincent. 2010. "Noncooperative Foundations of Stable Sets in Voting Games." Games and Econ Behavior 70 (2): 48893.

Anesi, Vincent, and Daniel J. Seidmann. 2015. "Bargaining in Standing Commit tees with an Endogenous Default." Rev Econ Studies 82 (3): 82567.

Azzimonti, Marina. 2011. "Barriers to Investment in Polarized Societies." A.E.R. 101 (5): 21822204

Bafumi, Joseph, and Michael C. Herron. 2010. "Leapfrog Representation and Extremism: A Study of American Voters and Their Members in Congress." American Polit. Sci. Rev. 104:519 42. 
Bai, Jinhui H., and Roger Lagunoff. 2011. "On the Faustian Dynamics of Policy and Political Power." Rev. Econ. Studies 78 (1): 1748.

Baron, David. 1996. “A Dynamic Theory of Collective Goods Programs.” American Polit. Sci. Rev. 90 (2): 31630.

Baron, David, and Renee Bowen. 2013. "Dynamic Coalitions." Manuscript, Grad. School Bus., Stanford Univ.

Baron, David, Daniel Diermeier, and Pohan Fong. 2012. "A Dynamic Theory of Parliamentary Democracy.” Econ. Theory 49 (1): 70338.

Baron, David, and John A. Ferejohn. 1989. "Bargaining in Legislatures.” Ameri can Polit. Sci. Rev. 83 (4): 11811206.

Baron, David, and Michael Herron. 2003. "A Dynamic Model of Multidimen sional Collective Choice." In Computational Models of Political Economy, edited by Ken Kollman, John H. Miller, and Scott E. Page, 13 47. Cambridge, MA: MIT Press.

Baron, David, and Ehud Kalai. 1993. "The Simplest Equilibrium of a Majority Rule Division Game.” I. Econ. Theory 61 (2): 290301.

Battaglini, Marco, and Stephen Coate. 2007. "Inefficiency in Legislative Policy making: A Dynamic Analysis." A.E.R. 97 (1): 11849.

. 2008. "A Dynamic Theory of Public Spending, Taxation, and Debt." A.E.R. 98 (1): 20136.

Bernheim, Douglas, Antonio Rangel, and Luis Rayo. 2006. "The Power of the Last Word in Legislative Policy Making." Econometrica 74 (5): 116190.

Besley, Timothy, and Stephen Coate. 1998. "Sources of Inefficiency in a Repre sentative Democracy: A Dynamic Analysis.” A.E.R. 88 (1): 13956.

Bhaskar, V., George J. Mailath, and Stephen Morris. 2013. "A Foundation for Markov Equilibria in Sequential Games with Finite Social Memory." Rev. Econ. Studies 80 (3): 100226.

Bowen, Renee, Ying Chen, and Hulya Eraslan. 2014. "Mandatory versus Discre tionary Spending: The Status Quo Effect.” A.E.R. 104 (10): 294174.

Bowen, Renee, Ying Chen, Hulya Eraslan, and Jan Zapal. 2014. "Efficiency of Flex ible Budgetary Institutions.” Manuscript, Grad. School Bus., Stanford Univ.

Bowen, Renee, and Zaki Zahran. 2012. "On Dynamic Compromise." Games and Econ. Behavior 76 (2): 391419.

Brady, David W., and Craig Volden. 2006. Revolving Gridlock: Politics and Policy from Jimmy Carter to George W. Bush. 2nd ed. Boulder, CO: Westview.

Calabresi, Guido. 1982. A Common Law for the Age of Statutes. Cambridge, MA: Har vard Univ. Press.

Cho, Seok ju. 2012. "The Dynamics of Parliamentary Bargaining and the Vote of Confidence." Manuscript. https://drive.google.com/file/d/0B3p4mmebrd 1U04zMXRCZ3RIbEE/view.

Coate, Stephen, and Stephen Morris. 1999. "Policy Persistence." A.E.R. 89:1327 36.

Dal Bó, Ernesto. 2006. "Committees with Supermajority Voting Yield Commit ment with Flexibility." I. Public Econ. 90:573 99.

Diermeier, Daniel, and Pohan Fong. 2011. "Legislative Bargaining with Recon sideration." O.J.E. 126 (2): 94785.

Drazen, Allan. 2002. Political Economy in Macroeconomics. Princeton, NJ: Princeton Univ. Press.

Duggan, John, and Jean G. Forand. 2013. "Markovian Elections." Manuscript, Dept. Polit. Sci., Univ. Rochester.

Duggan, John, and Tasos Kalandrakis. 2012. "Dynamic Legislative Policy Mak ing." I. Econ. Theory 147 (5): 165388. 
Dziuda, Wioletta, and Antoine Loeper. 2012. "Dynamic Collective Choice with Endogenous Status Quo.” Manuscript, Kellogg School Management, North western Univ.

. 2015. "Voting Rules in a Changing Environment." Manuscript, Kellogg School Management, Northwestern Univ.

Eilenberg, Samuel, and Deane Montgomery. 1946. "Fixed Point Theorems for Multi valued Transformations." American J. Math. 68:214 22.

Fernandez, Raquel, and Dani Rodrik. 1991. "Resistance to Reform: Status Quo Bias in the Presence of Individual Specific Uncertainty." A.E.R. 81:1146 55.

Fiorina, Morris P., and Samuel J. Abrams. 2008. "Political Polarization in the American Public." Ann. Rev. Polit. Sci. 11:563 88.

Fiorina, Morris P., Samuel J. Abrams, and Jeremy C. Pope. 2006. Culture War? The Myth of a Polarized America. 2nd ed. New York: Pearson Longman.

Friedman, John N., and Richard T. Holden. 2009. "The Rising Incumbent Reelec tion Rate: What's Gerrymandering Got to Do with It?" I. Politics 71:593 611.

Gale, William G., and Peter R. Orszag. 2003. "Sunsets in the Tax Code.” Tax Notes 99:1553 61 .

Gersen, Jacob E. 2007. "Temporary Legislation." Univ. Chicago Law Rev. 74 (1): 24798.

Glomm, Gerhard, and B. Ravikumar. 1995. "Endogenous Public Policy and Mul tiple Equilibria.” European I. Polit. Econ. 11 (4): 65362.

Gradstein, Mark. 1999. "Optimal Taxation and Fiscal Constitution." I. Public Econ. 72:471 85 .

Hayes, Michael T. 1992. Incrementalism and Public Policy. New York: Longman.

Jefferson, Thomas. [1789] 1958. Letter from Thomas Jefferson to James Madi son, September 6. In The Papers of Thomas Jefferson, vol. 15, edited by Julian P. Boyd, 392 97. Princeton, NJ: Princeton Univ. Press.

Kalandrakis, Tasos. 2004. "A Three Player Dynamic Majoritarian Bargaining Game." I. Econ. Theorv 116 (2): 294322.

. 2010. "Minimum Winning Coalitions and Endogenous Status Quo." Internat. J. Game Theory 39 (4): 61743.

Krasa, Stefan, and Mattias Polborn. 2014. "Policy Divergence and Voter Polariza tion in a Structural Model of Elections." I. Law and Econ. 57 (1): 3176.

Krehbiel, Keith. 1991. Information and Legislative Organization. Ann Arbor: Univ. Michigan Press.

. 1999. "Pivotal Politics: A Refinement of Nonmarket Analysis for Voting Institutions." Bus. and Politics 1 (1): 6381.

Krusell, Per, and Jose Victor Rios Rull. 1996. "Vested Interests in a Positive The ory of Stagnation and Growth.” Rev. Econ. Studies 63 (2): 30129.

.1999. "On the Size of the U.S. Government: Political Economy in the Neo classical Growth Model." A.E.R. 89 (5): 115681.

Kysar, Rebecca M. 2011. "Lasting Legislation." Univ. Pennsylvania Law Rev. 159: 100752.

Levit, Mindy R., and Andrew D. Austin. 2014. Mandatory Spending since 1962. Washington, DC: Congressional Res. Service.

Lindblom, Charles. 1959. "The Science of Muddling Through." Public Admin. Rev. 19:79 88.

Lowi, Theodore J. 1969. The End of Liberalism: Ideology, Policy, and the Crisis of Public Authority. New York: Norton.

McCarty, Nolan, Keith T. Poole, and Howard Rosenthal. 2006. Polarized America: The Dance of Ideology and Unequal Riches. Cambridge, MA: MIT Press. 
Meyn, Sean P., and Richard L. Tweedie. 1993. Markov Chains and Stochastic Stabil ity. London: Springer Verlag.

Mooney, Chris. 2004. “A Short History of Sunsets.” Legal Affairs, January February.

Persson, Torsten, and Lars E. O. Svensson. 1989. "Why a Stubborn Conservative Would Run a Deficit: Policy with Time Inconsistent Preferences." O.J.E. 104:325 45 .

Poole, Keith T., and Howard Rosenthal. 1997. Congress: A Political Economic History of Roll Call Voting. New York: Oxford Univ. Press.

Posner, Eric, and Adrian Vermeule. 2002. "Legislative Entrenchment: A Reap praisal." Yale Law I. 111:1665 1705.

Reny, Phil J. 2011. "On the Existence of Monotone Pure Strategy Equilibria in Bayesian Games.” Econometrica 79 (2): 499553.

Riboni, Alessandro, and Francisco J. Ruge Murcia. 2008. "The Dynamic (In)effi ciency of Monetary Policy by Committee." I. Monev. Credit. and Banking 40 (5): 100132.

. 2010. "Monetary Policy by Committee: Consensus, Chairman Domi nance or Simple Majority?” O.J.E. 125:363 416.

Richter, Michael. 2014. "Fully Absorbing Dynamic Compromise." $\underline{\text { I. Econ. Theory }}$ 152:92 104.

Romer, Thomas, and Howard Rosenthal. 1978. "Political Resource Allocation, Controlled Agendas, and the Status Quo." Public Choice 33 (4): 2743.

Strulovici, Bruno. 2010. "Learning While Voting: Determinants of Collective Ex perimentation." Econometrica 78 (3): 93371.

Wildavsky, Aaron. 1992. The New Politics of the Budgetary Process. 2nd ed. New York: HarperCollins.

Zapal, Jan. 2011a. "Explicit and Implicit Status Quo Determination in Dynamic Bargaining: Theory and Application to FOMC Directive." Manuscript, CERGE EI, Prague.

. 2011b. "Simple Equilibria in Dynamic Bargaining Games over Policies." Manuscript, CERGE EI, Prague. 